\title{
Besteht ein Zusammenhang zwischen der Gender-Diversity und Überwachungseffektivität des Aufsichtsrats? Eine empirische Analyse deutscher börsennotierter Unternehmen
}

\author{
Franziska Handschumacher (D) - Willi Ceschinski
}

Eingegangen: 30. September 2019 / Angenommen: 23. Mai 2020 / Online publiziert: 7. Juni 2020

(C) Der/die Autor(en) 2020

Zusammenfassung Vor dem Hintergrund gesetzlicher Novellierungen und des gesellschaftlichen Rufs nach mehr geschlechterspezifischer Gleichberechtigung untersucht der vorliegende Beitrag den Zusammenhang zwischen der Gender-Diversity im Aufsichtsrat und seiner Überwachungseffektivität. Auf Grundlage der Agency Theorie und der Resource Dependence Theorie sowie anhand mehrerer unternehmensfixen Panelregressionen testen wir die vorstehende Verbindung. Der zugrundeliegende Paneldatensatz umfasst ca. 1500 Aufsichtsräte von 132 Unternehmen, die in den Jahren 2015-2017 im HDAX und SDAX gelistet waren (396 Unternehmensjahre). Gender-Diversity wird durch verschiedene Diversitäts-Kennzahlen gemessen und Überwachungseffektivität mittels übermäßiger Vorstandsvergütung operationalisiert. Die Ergebnisse deuten darauf hin, dass eine höhere Repräsentanz von Frauen im Aufsichtsrat (Aktionärsvertreter und gesamter Aufsichtsrat) mit einer geringeren übermäßigen Vergütung und folglich einer besseren Überwachungseffektivität assoziiert ist. Dagegen können wir keinen Zusammenhang zwischen der GenderDiversity der Arbeitnehmervertreter bzw. der Mitglieder im Vergütungsausschuss und der vergütungsbezogenen Überwachungseffektivität feststellen. Nachdem sich die internationale Forschung bereits vereinzelt mit der Thematik auseinandergesetzt hat, wird erstmalig im Kontext des dualistischen Corporate Governance-Systems getestet, inwiefern Geschlechtervielfalt im Aufsichtsrat und vergütungsbezogene Überwachungseffektivität miteinander verknüpft sind. Der Beitrag legt hierzu dar, dass eine höhere Gender-Diversity, unabhängig von sozialpolitischen Überlegungen, vor-

F. Handschumacher $(\bowtie) \cdot$ W. Ceschinski

Institut für Wirtschaftsprüfung und Steuerwesen, Universität Hamburg,

Von-Melle-Park 5, 20146 Hamburg, Deutschland

E-Mail: Franziska.Handschumacher@uni-hamburg.de

W. Ceschinski

E-Mail: Willi.Ceschinski@uni-hamburg.de 
teilhaft für die Corporate Governance von Unternehmen sein kann und trägt somit zur Diskussion über die Wirkung der gesetzlichen Geschlechterquote bei.

Schlüsselwörter Gender-Diversity · Corporate Governance · Aufsichtsrat · Überwachungseffektivität · Vorstandsvergütung · Mitbestimmung

JEL G34 $\cdot \mathrm{G} 38 \cdot \mathrm{J} 16 \cdot \mathrm{M} 12 \cdot \mathrm{M} 51 \cdot \mathrm{C} 12 \cdot \mathrm{J} 16 \cdot \mathrm{J} 23 \cdot \mathrm{J} 31$

\title{
Is There a Link Between Gender Diversity and Supervisory Board's Monitoring Effectiveness? An Empirical Analysis of German Listed Companies
}

\begin{abstract}
Against the background of legal amendments and society's call for more gender-specific equality, this article examines the relationship between gender diversity in the supervisory board and its monitoring effectiveness. We test the aforementioned relationship based on agency theory and resource dependence theory and on the basis of several company-fixed panel regressions. The underlying panel data comprises 1500 supervisory boards of 132 companies listed in HDAX and SDAX between 2015 and 2017 (396 company years). Gender diversity is operationalized through different diversity metrics, and monitoring effectiveness is measured by excessive board compensation. The results indicate that a higher representation of women on the supervisory board (shareholder representatives and all members) is accompanied by lower excessive compensation and thus better monitoring effectiveness. In contrary, we cannot find a relationship between gender diversity of employee representatives or all members of the compensation committee and monitoring effectiveness. After international research has already dealt occasionally with this context, it is now being tested for the first time in the context of the dualistic corporate governance-system to what extent gender diversity in the supervisory board and the remuneration-related monitoring effectiveness are linked. Our paper argues that greater gender diversity, regardless of socio-political considerations, can be beneficial for companies' corporate governance and thus contributes to the discussion about the impact of the statutory gender quota.
\end{abstract}

Keywords Gender Diversity · Corporate Governance · Supervisory Board · Monitoring Effectiveness · Executive Board Remuneration · Co-Determination

\section{Einführung}

Im Mai 2015 trat in Deutschland das Gesetz für die gleichberechtigte Teilhabe von Frauen und Männern an Führungspositionen (FüPoG) in Kraft. Mit dem Ziel den Frauenanteil in Führungspositionen zu steigern, haben börsennotierte und voll mitbestimmungspflichtige Aktiengesellschaften seit 2016 bei der Neubesetzung ihrer Aufsichtsratspositionen die gesetzliche Quote von 30\% für das jeweils unterrepräsentierte Geschlecht zu berücksichtigen. Bei Nichtbesetzung offener Aufsichtsratspositionen durch einen Vertreter des bisher unterrepräsentierten Geschlechts haben 
die vakanten Stellen unbesetzt zu bleiben ( $\$ 96$ Abs. 2 AktG). Darüber hinaus müssen Unternehmen Zielgrößen und Fristen für den Frauenanteil im Aufsichtsrat, im Vorstand sowie in den beiden Führungsebenen unterhalb des Vorstands festlegen und diese in der Erklärung zur Unternehmensführung offenlegen. Sofern der Frauenanteil bei Festlegung der Zielgröße unter $30 \%$ liegt, darf diese den aktuellen Frauenanteil nicht unterschreiten (§76 Abs. 4 AktG und $\$ 111$ Abs. 5 AktG i. V.m. §289f Abs. 2 Nr. 4 HGB). Diese Vorgabe führte in der Praxis dazu, dass die Zielgröße z. T. mit $0 \%$ angegeben wurde, um diese künftig nicht mehr unterschreiten zu können (Maaß 2016).

Mit der Einführung der Geschlechterquote folgt Deutschland dem Beispiel einiger europäischer Länder, wie Norwegen oder Spanien, die bereits 2003 bzw. 2007 gesetzliche Geschlechterquoten beschlossen. In 2018 wurde in Kalifornien und damit erstmalig in den USA eine Geschlechterquote eingeführt (von Meyerinck et al. 2020). Das Thema der Gender-Diversity ist somit aktuell wie nie zuvor. Während sich bereits einige Studien mit dem Zusammenhang zwischen Gender-Diversity und dem finanziellen sowie nichtfinanziellen Unternehmenserfolg beschäftigten, bleiben weitere Unternehmensmerkmale (zur Corporate Governance), insbesondere im deutschensprachigen Forschungsraum, bisher weitestgehend unberücksichtigt (Velte 2017a). Darüber hinaus wurden vor allem in den USA und Skandinavien zahlreiche empirische Studien zur ökonomischen Bedeutung weiblicher Führungspersonen im monistischen Corporate Governance-System publiziert, wohingegen in Deutschland weiterhin Forschungsbedarf auf diesem Gebiet besteht (Momtaz 2014; Reinwald et al. 2015; Handschumacher et al. 2018). Empirische Evidenzen im Kontext des monistischen Corporate Governance-Systems lassen sich aufgrund der unterschiedlichen Rahmenbedingungen nur eingeschränkt auf das dualistische System übertragen (Dienes und Velte 2016). Das in Deutschland vorherrschende dualistische System, welches sich durch eine strikte Trennung und daraus resultierende gegenseitige Überwachung von Unternehmensführung und -überwachung auszeichnet, unterscheidet sich damit grundlegend vom monistischen Modell (Winkler 2018). Des Weiteren zeichnet sich das deutsche Corporate Governance-System durch die Mitbestimmung der Arbeitnehmer im Aufsichtsrat aus, womit sich das System auch dahingehend von anderen Modellen unterscheidet (Raabe 2011). Vor diesem Hintergrund präsentiert der vorliegende Beitrag zunächst, inwiefern sich die Repräsentanz weiblicher Aufsichtsräte in den letzten Jahren entwickelt hat. Wir setzen sodann an den dargelegten Forschungslücken an, indem wir den Zusammenhang von Gender-Diversity im Aufsichtsrat und der Überwachungseffektivität des Kontrollgremiums im Kontext des dualistischen Systems der Unternehmensverfassung empirisch untersuchen.

Unter Berücksichtigung, dass der Aufsichtsrat nur mittelbar in die Unternehmensstrategie involviert ist und sich Prozesse innerhalb des Gremiums kaum beobachten und operationalisieren lassen, ist der Einfluss des Kontrollgremiums auf den Unternehmenserfolg in der Forschung umstritten (Schulten 2013; Kirsch 2018). Eine empirische Verknüpfung von Gender-Diversity und dem Unternehmenserfolg setzt voraus, dass sich die Qualität der Corporate Governance im Allgemeinen und die Arbeit des Aufsichtsrats im Speziellen zum einen auf den Unternehmenswert auswirken und zum anderen anhand geeigneter Erfolgskennzahlen ablesen lassen. Der Unternehmenserfolg wird jedoch durch zahlreiche Faktoren beeinflusst, sodass die 
Empirie nur eingeschränkt von diesem auf die Arbeitsweise des Aufsichtsrats schließen kann. Hinzu kommt, dass Endogenitätsprobleme keine eindeutige Aussage über den Kausalzusammenhang zwischen Gender-Diversity und der Unternehmensperformance zulassen (Winkler 2018). Aus diesem Grund bezieht sich der vorliegende Beitrag direkt auf die Tätigkeit des Aufsichtsrats. Auf Grundlage der Agency Theorie beurteilen wir die Effektivität des Kontrollorgans danach, inwiefern es ihm gelingt, den Vorstand zu überwachen und die aus der Trennung von Eigentum und Kontrolle entstandenen Agency-Kosten zu verringern. Aufgrund der schwierigen Quantifizierbarkeit der Aufsichtsratstätigkeit, verwendet wir ein etabliertes Surrogat der Überwachungseffektivität, indem wir diese, Core et al. (1999) sowie Winkler und Behrmann (2019) folgend, anhand der übermäßigen Vorstandsvergütung (excess pay) approximieren. Bei Verwendung der übermäßigen Vergütung als Surrogat der Überwachungseffektivität gehen wir davon aus, dass Vorstände, bei einer ineffizienten Überwachung durch den Aufsichtsrat, eine höhere Vergütung erhalten, die nicht an ökonomische Determinanten geknüpft ist (Coles et al. 2014). Die Ineffizienz des Aufsichtsrats wird bisweilen auf Interessenkonflikte oder Abgelenktheit durch hohe Vernetzung und Mandatskumulierungen der Aufsichtsratsmitglieder oder unzureichende Expertise des Gremiums zurückgeführt (Handschumacher et al. 2019; John und Senbet 1998). Ein Vorteil der Operationalisierung der Überwachungseffektivität durch die übermäßige Vorstandsvergütung liegt darin, dass excess pay als quantitatives Ergebnis der Aufsichtsratstätigkeit in Bezug auf die Vorstandsvergütung erfasst werden kann ( $\$ 87 \mathrm{AktG})$, wodurch Endogenitätsprobleme (bspw. Reverse Causality) zwar nicht ausgeschlossen, jedoch reduziert werden können (Seo 2017; Winkler und Behrmann 2019).

Die Beziehung zwischen Gender-Diversity und übermäßiger Vergütung wird anhand eines händisch erhobenen Datensatzes geschätzt, welcher 132 börsennotierte deutsche Unternehmen mit ca. 1500 Aufsichtsräten der Geschäftsjahre 2015-2017 umfasst (396 Unternehmensjahre). Dazu schätzen wir mehrere Regressionsanalysen mit fixen Unternehmenseffekten (Fixed-Effects-Modelle) sowie fünf DiversityKennzahlen als unabhängige und excess pay als abhängige Variable. Die Ergebnisse deuten auf einen inversen Zusammenhang zwischen der Geschlechtervielfalt im Aufsichtsrat und der übermäßigen Vergütung hin. Der Zusammenhang ist bei Berücksichtigung der Geschlechterheterogenität der Aktionärsvertreter und des gesamten Aufsichtsrats, dagegen nicht hinsichtlich der Arbeitnehmervertreter und des Vergütungsausschusses statistisch nachweisbar. Als Vergütungsausschuss ist hier und im Folgenden der Ausschuss des Aufsichtsrats zu verstehen, der für die Festsetzung der Vergütung verantwortlich ist, auch wenn die Selbstbezeichnung dieser Ausschüsse ggf. abweichend lautet. Sodann untersuchen wir den nichtlinearen Zusammenhang zwischen weiblichen Aufsichtsrätinnen und der übermäßigen Vergütung und stellen fest, dass der dargelegte Zusammenhang stärker ist, wenn genau eine Frau oder mindestens vier Frauen im Aufsichtsrat vertreten sind. Damit lassen unsere empirischen Befunde vermuten, dass die Geschlechterquote, in Hinblick auf die vergütungsbezogene Überwachungseffektivität, nicht zum Nachteil guter Corporate Governance eingeführt wurde. Vor diesem Hintergrund leistet die Untersuchung einen Beitrag zur aktuellen Diskussion über die Rolle von Frauen in Führungspositionen und ihren Beitrag zur Qualität der Corporate Governance. Auch unabhängig gesetzlicher 
Vorschriften, liefert die Studie Implikationen für den Aufsichtsrat bei Planung seiner Zusammensetzung.

Der Beitrag gliedert sich wie folgt: Der Einführung folgend werden im zweiten Kapitel anhand der Prinzipal Agenten Theorie, Resource Dependence Theorie und Critical Mass Theorie die empirischen Analysen theoretisch fundiert und die Hypothesen formuliert. Kapitel drei erläutert das Design der im vierten Kapitel dargestellten empirischen Untersuchung. Die Präsentation der Empirie beginnt mit der deskriptiven Statistik, auf welche die Korrelations- und Regressionsanalysen folgen. Der Beitrag endet mit einem Fazit und Ausblick.

\section{Theoretische Fundierung, Forschungstand und Hypothesenableitung}

\subsection{Prinzipal Agenten Theorie, Resource Dependence Theorie und Critical Mass Theorie}

Die Prinzipal Agenten Theorie und Resource Dependence Theorie gelten als führende Erklärungsansätze in der Corporate Governance-Forschung (Drobetz et al. 2004; Schmidt und Brauer 2005; Eulerich et al. 2014).

Die Prinzipal Agenten Theorie (Agency Theory) evaluiert Vertragsbeziehungen, in denen ein Auftraggeber (Prinzipal) Entscheidungsbefugnis an einen Auftragnehmer (Agent) delegiert (Fama und Jensen 1983; Hochhold und Rudolph 2009). Da der Agent zwar Entscheidungsträger ist, jedoch nicht das aus seinem Handeln resultierende Risiko trägt, führt dies zu Agency-Kosten zu Lasten des Prinzipals (Jensen und Meckling 1976). Übertragen auf das dualistische Corporate Governance-System fungiert der Vorstand als Agent der Stakeholder, die ihm die Führung des Unternehmens übertragen. Durch die Trennung von Eigentum und Kontrolle wird dem Vorstand ein gewisser Handlungsspielraum eingeräumt, den dieser zur Durchsetzung seiner eigenen Interessen ausnutzen kann (Jensen und Meckling 1976; Hochhold und Rudolph 2009; Prinz 2012). Opportunistische Verhaltensweisen des Vorstands äußern sich bspw. darin, dass dieser nach höherer Macht oder Vergütung strebt (Tebben 2011; Freidank 2012). Zur Kontrolle des Vorstands dienen im Unternehmen verschiedene Corporate Governance-Mechanismen mit der Zielsetzung das eigennützige Verhalten des Vorstands und damit die Agency-Kosten zu minimieren. Im dualistischen Corporate Governance-System fungiert der Aufsichtsrat als Kontrollorgan, welches durch die Unternehmenseigentümer implementiert wird (Oehmichen 2011). In diesem Punkt unterscheidet sich das dualistische vom monistischen System, welches überwiegend im angelsächsischen Raum vertreten ist und sich durch ein einheitliches Führungs- und Überwachungsorgan (board) auszeichnet (Eulerich und van Uum 2017). Eine Aufgabe des Aufsichtsrats besteht darin, diskretionäre Handlungsspielräume des Vorstands einzuschränken. Inwiefern diese Aufgabe effektiv erfüllt wird, kann insofern beurteilt werden, in welchem Umfang der Aufsichtsrat opportunistische Verhaltensweisen des Vorstands minimiert.

Der Resource Dependence Theorie zufolge wird der Erfolg eines Unternehmens von seinem Zugang zu kritischen Ressourcen determiniert (Hillman et al. 2000). Zu den erfolgskritischen Ressourcen gehören z. B. Rohstoffe, Finanz- und Humankapi- 
tal, Informationen etc., die Unternehmen von ihrer Umwelt beziehen. Damit stehen Unternehmen in reziproker Abhängigkeit zu ihren Ressourcenquellen, welche bspw. andere Unternehmen, Kapitalgeber, der Staat oder der Arbeitsmarkt sein können (Pfeffer und Salancik 1978; Brunner 2009; Fessler 2013). Gelingt es Unternehmen sich Zugang zu ihren erfolgskritischen Ressourcen zu verschaffen, kann ihre Abhängigkeit gesenkt werden, woraus reduzierte Unsicherheiten und Transaktionskosten resultieren (Boyd 1990; Hillman und Dalziel 2003). Die Corporate GovernanceForschung sieht in diesem Zusammenhang den Aufsichtsrat als strategisches Instrument, um kritische Ressourcen in das Unternehmen zu integrieren (Hillman et al. 2000; Nicholson und Kiel 2007)

Anhand der Prinzipal Agenten Theorie und der Resource Dependence Theorie argumentiert die Corporate Governance-Forschung, dass der Aufsichtsrat zur Kontrolle des Vorstands über weitreichendes Humankapital verfügen sollte, welches mitunter Erfahrung, Wissen und Reputation umfasst (Hillman et al. 2000; Cumming et al. 2015). Die Wissenschaft plädiert daher dafür, dass diese Anforderungen durch eine heterogene Zusammensetzung des Gremiums erreicht werden können (Carter et al. 2003; Hillman und Dalziel 2003; Cumming et al. 2015; Fehre und Spiegelhalder 2017). Diversifiziertes Humankapital soll neue Perspektiven eröffnet und Diskussionen im Gremium anregen (Rose 2007; Handschumacher et al. 2018). Dabei spielen weibliche Führungspersönlichkeiten, in den zumeist männerdominierten Gremien, eine einflussreiche Rolle. Einige Studien belegen, dass weibliche Führungskräfte andere Verhaltensweisen an den Tag legen als männliche (Cumming et al. 2015). Zudem unterscheiden sich Frauen und Männer durch ihr Wissen, ihre Erfahrung und ihre Ansichten (Fehre und Spiegelhalder 2017). Beide Aspekte schlagen sich wiederum in den strategischen Entscheidungen des Aufsichtsrats nieder und beeinflussen somit seine Überwachungseffektivität (Cumming et al. 2015; Reinwald et al. 2015).

Hinsichtlich geschlechterspezifischer Verhaltensweisen wird in der Forschung häufig die Critical Mass Theorie von Kanter (1977) angebracht. Die Theorie analysiert Gruppeninteraktionen anhand von vier unterschiedlichen Gruppen mit ausschließlich männlichen Mitgliedern (uniform groups), hauptsächlich männlichen Mitgliedern (skewed groups), relativ ausgeglichen Geschlechterverhältnissen (tilted groups) und ausgeglichenen Geschlechterverhältnissen (balanced groups). Uniforme Gruppen, in denen wenige Frauen (token) von vielen Männern (>80\%) dominiert werden, können zwei mögliche Probleme hervorrufen. Auf der einen Seite können Frauen stereotypisiert werden und Geschlechterunterschiede in den Vordergrund rücken. Dies hat zur Folge, dass Frauen ausgeschlossen werden, sich gegenüber ihren männlichen Kollegen nicht behaupten und keinen Einfluss auf Entscheidungen des Gremiums ausüben können. Auf der anderen Seite kommt es vor, dass Unterschiede heruntergespielt werden und Frauen Verhaltensweisen ihrer männlichen Kollegen assimilieren, womit die zuvor genannten Vorteile der Gruppenheterogenität ausbleiben, da das assimilierte Verhaltens keine neue Vielfalt in den Aufsichtsrat bringt (Joecks et al. 2013). Beide Phänomene führen dazu, dass die Arbeitsleistung derartiger Gruppen unter die Leistungsfähigkeit rein männlicher Gremien fällt, da Diversität keinen neuen Input liefert und die Kosten in diesen Fällen den geringen Nutzen übersteigen. Einige Autoren stützen sich daher auf die Annahme, dass Aufsichtsrätinnen erst ab einem bestimmten Frauenanteil (critical mass) die Möglichkeit 
haben, ihre Interessen und Kompetenzen einzubringen (Konrad et al. 2008; Joecks et al. 2013).

\subsection{Literaturübersicht und Hypothesenbildung}

Seit einigen Jahren wächst das Interesse der nationalen und internationalen Forschung am Thema Corporate Governance im Allgemeinen und der Gender-Diversity in Führung- und Kontrollgremien im Speziellen (Bozhinov et al. 2019). Laut Kirsch (2018) wurden zwischen 1981 und 2016 insgesamt 310 Studien zur GenderDiversity im Vorstand und Aufsichtsrat publiziert, wobei etwa die Hälfte dieser aus den letzten drei Jahren der Betrachtung (2013-2016) stammt. Dabei konzentrieren sich die Studien insbesondere auf die Untersuchung des Zusammenhangs von Gender-Diversity und der finanziellen sowie der sozialen und ethischen Performance von Unternehmen (Kirsch 2018). Nur untergeordnet haben vorangegangene Publikationen die Tätigkeit, und dementsprechend die Effektivität, des Kontrollgremiums selbst betrachtet (Lucas-Pérez et al. 2015). Darüber hinaus steht das international etablierte monistische Board-System im Vordergrund der Untersuchungen, während das in Deutschland vorherrschende dualistische System in diesem Kontext bislang weniger Beachtung fand (Handschumacher et al. 2018). Des Weiteren sind ältere Forschungsergebnisse mit Einschränkungen zu berücksichtigen, zumal ihr Datensatz einen geringen Grad der Gender-Diversity aufweist und empirische Zusammenhänge somit weniger ausgeprägt und nachweisbar sind (Boerner et al. 2012).

Obwohl zum Zusammenhang von Frauen im Aufsichtsrat bzw. Vorstand und der (nicht-) finanziellen Unternehmensperformance einige Studien existieren, herrscht hinsichtlich ihrer Vor- und Nachteiligkeit Dissens. Bspw. stellen Reguera-Alvarado et al. (2017), Gulamhussen und Santa (2015), Campbell und Mínguez-Vera (2008) sowie Carter et al. (2003) einen positiven Zusammenhang zwischen Frauen im Führungs- und Kontrollgremium (Board) und dem finanziellen Unternehmenserfolg fest. Dem widersprechend können die Ergebnisse der Studien von Hassan und Marimuthu (2016), Chapple und Humphrey (2014), Rose (2007) sowie Randøy et al. (2006) keine signifikante Verbindung von weiblichen Mitgliedern des Gremiums und dem Unternehmenserfolg aufdecken. Schließlich postulieren Abdullah et al. (2016), Ahern und Dittmar (2012) und Shrader et al. (1997), dass Frauen im Board mit einem geringeren finanziellen Unternehmenserfolg einhergehen. Neben der finanziellen Performance untersuchen weitere Studien den Zusammenhang zwischen Frauen in Führungs- und Kontrollorganen und der Corporate Social Responsibility (CSR) bzw. dem CSR-Reporting von Unternehmen und präsentieren eine überwiegend positive Verbindung. Exemplarisch legen Boulouta (2013), Harjoto et al. (2015), Bear et al. (2010) sowie Dienes und Velte (2016) eine positive Assoziation mit der CSR bzw. dem CSR-Reporting offen, während die Studie von Manita et al. (2018) keinen signifikanten Zusammenhang vermuten lässt.

Darüber hinaus analysieren einige Veröffentlichungen den Zusammenhang zwischen weiblichen Vorständen sowie Aufsichtsräten und weiteren Unternehmensparametern. Hierbei konstatieren bspw. Lenard et al. (2014), dass eine höhere GenderDiversity im Board mit einer niedrigeren Volatilität der Aktienrendite zusammenhängt, welche in der Studie als Proxy des Unternehmensrisikos verwendet wird. 
Daneben lässt die Untersuchung von Chen et al. (2016) vermuten, dass ein höherer Frauenanteil im Board mit einer geringeren Anzahl und Größe von Unternehmensakquisitionen einhergeht. Velte (2017b) weist darauf hin, dass der relative Anteil von Frauen im Aufsichtsrat mit höheren Prüfungshonoraren verknüpft ist. Hieraus wird auf eine bessere Effektivität der Aufsichtsratstätigkeit geschlossen, die sich im Einsatz von erhöhten zeitlichen und fachlichen Ressourcen des Abschlussprüfers niederschlägt.

Ferner wurden im internationalen Kontext einige Untersuchungen über den $\mathrm{Zu}$ sammenhang von Gender-Diversity im Aufsichtsrat und der Überwachungseffektivität in Bezug auf die Vergütung des Vorstands veröffentlicht. Initial untersuchen Adams und Ferreira (2009) den Wirkungszusammenhang zwischen Frauen im Aufsichtsrat und dem Anteil der variablen Vergütung an der Gesamtvergütung als ProxyVariable guter Corporate Governance. Der Frauenanteil im Aufsichtsrat ist hier positiv und signifikant mit dem Anteil der variablen Vergütung verknüpft, steht jedoch in keinem empirisch nachweisbaren Verhältnis zur gesamten Vorstandsvergütung. Anhand dieser Befunde vermuten die Autoren einen vorteilhaften Effekt weiblicher Aufsichtsräte auf die Corporate Governance der Unternehmen. Auf dieser Studie aufbauend, gehen Lucas-Pérez et al. (2015) der Frage nach, inwiefern ein größerer Frauenanteil in spanischen Boards mit einem höheren Anteil der erfolgsabhängigen Vorstandsvergütung und damit einer besseren Überwachungsqualität einhergeht. Die Überwachungseffektivität approximieren die Autoren ebenfalls anhand der durchschnittlichen variablen Vergütung im Vorstand sowie dem Anteil der variablen Vergütung an der Gesamtvorstandsvergütung. Analog dazu bekräftigen Baixauli-Soler et al. (2016), dass Gender-Diversity und der Anteil der variablen Vorstandsvergütung zur Gesamtvergütung in einem positiven Zusammenhang zueinanderstehen. Des Weiteren zeigen die empirischen Ergebnisse, dass dieser Zusammenhang durch eine dominante Eigentümerstruktur abgeschwächt wird. Wie auch Adams und Ferreira (2009) sowie Lucas-Pérez et al. (2015), beobachten Bugeja et al. (2016) nicht nur den gesamten Aufsichtsrat, sondern explizit den Zusammenhang der GenderDiversity im Vergütungsausschuss und der übermäßigen Vorstandsvergütung. Ihren empirischen Ergebnissen zur Folge, besteht kein Wirkungszusammenhang von Gender-Diversity im gesamten Aufsichtsrat und der Vorstandsvergütung, während jedoch die Zusammensetzung des Vergütungsausschusses signifikant mit der Vorstandsentlohnung zusammenhängt. Dem widersprechend zeigen Strobl et al. (2016), dass der Frauenanteil im Vergütungsausschuss in keinem signifikanten Zusammenhang zur performanceabhängigen Vorstandsvergütung steht. Entgegen der vorangegangenen Studien konstatieren Benkraiem et al. (2017), dass der Frauenanteil im Führungsund Kontrollorgan (board) und die Vorstandsvergütung signifikant und positiv assoziiert sind. Jedoch finden auch sie empirische Evidenz für eine negative Verknüpfung von unabhängigen Aufsichtsrätinnen und der Vorstandsvergütung. Usman et al. (2018) wiederum demonstrieren empirische Evidenzen, dass geschlechterheterogene Vergütungsausschüsse die Gesamtvergütung des CEOs einschränken und die Verbindung zwischen der Vorstandsvergütung und der Unternehmensperformance (Pay-for-Performance-Sensitivität) chinesischer Unternehmen stärken. Darüber hinaus stehen Vergütungsausschüsse mit einem kritischen Anteil weiblicher Auf- 
sichtsräte in einem engeren Zusammenhang zur Vorstandsvergütung und Pay-forPerformance-Sensitivität als Ausschüsse mit nur einer Aufsichtsrätin.

Während im internationalen Kontext bereits einige Studien zur Gender-Diversity und vergütungsbezogenen Überwachungseffektivität veröffentlich wurden, fehlt es dazu bislang an Untersuchungen zum dualistischen Corporate Governance-System. Die Befunde der vorangegangenen Studien geben zwar Hinweise auf einen möglichen empirischen Zusammenhang, lassen sich aber - aufgrund abweichender Rahmenbedingungen - eingeschränkt auf das dualistische System der Unternehmensverfassung übertragen. Zum einen wird, im Gegensatz zum monistischen System, die Vorstandsvergütung durch den Aufsichtsrat determiniert und somit stärker kontrolliert (Kara 2010). Neben der Trennung von Führung und Kontrolle, wird das deutsche dualistische System zum anderen durch die Mitbestimmung der Arbeitnehmer charakterisiert. Dieser Aspekt ist insbesondere bei der Untersuchung zur Wirkung von Frauen im Aufsichtsrat nicht zu vernachlässigen, da die Repräsentanz weiblicher Aufsichtsräte vermehrt auf Seiten der Arbeitnehmervertreter ausgewiesen werden kann. In unserem Datensatz wird der Frauenanteil im Aufsichtsrat tatsächlich insbesondere durch Arbeitnehmerinnen determiniert, der durchschnittliche Anteil liegt hier für 2017 bei 27,2\%, wohingegen der Anteil weiblicher Aktionärsvertreter $24,5 \%$ entspricht. Ferner wird angebracht, dass Arbeitnehmervertreter nur gering in die Entscheidungsfindung des Gremiums involviert werden, da wichtige Beschlüsse (bspw. zur Festsetzung der Vorstandsvergütung) regelmäßig in Ausschüssen vorbereitet werden, in denen Arbeitnehmervertreter seltener vertreten sind (Raabe 2011). Im gleichen Kontext bringen Kritiker der gesetzlichen Geschlechterquote an, dass Frauen seltener in Ausschüsse berufen werden und somit ebenfalls keinen Einfluss auf strategische Entscheidungen des Aufsichtsrats haben (Oehmichen et al. 2010). Vor diesem Hintergrund sind auch der gesellschaftliche und sozialpolitische Kontext und sein Einfluss auf genderbezogene Diskussionen zu berücksichtigen (Boerner et al. 2012). Insbesondere entsteht vor dem Hintergrund der kürzlich eingeführten Geschlechterquoten Forschungsbedarf zu deren ökonomischer Wirkung. Aus diesem Grund strebt der Beitrag an, in die vorgelegte Forschungslücke einzudringen und betrachtet hierzu den Zusammenhang zwischen weiblichen Aufsichtsräten und der Wirkung der Tätigkeit des gesamten Kontrollorgans im Kontext der dualistischen Unternehmensverfassung. Dazu untersuchen wir empirisch, ob eine Verbindung zwischen dem Ausmaß der Gender-Diversity des Aufsichtsrats und seiner Überwachungseffektivität besteht. Anhand der Prinzipal Agenten Theorie argumentiert die Corporate Governance-Forschung, dass die Effektivität des Aufsichtsrats danach beurteilt werden kann, inwiefern er opportunistische Verhaltensweisen des Vorstands minimiert und mit den Stakeholder-Interessen in Einklang bringt. Aufsichtsräte können Vorstände beispielsweise durch die Festlegung der Vorstandsvergütung disziplinieren (Carter et al. 2003). Die Effizienz der Aufsichtsratskontrollen schlägt sich somit auch in einem anreizbasierten Vergütungssystem nieder (Faleye et al. 2011; Winkler 2018). Die Wirksamkeit des Vergütungssystems wird in der Forschung regelmäßig durch die Höhe der Vergütung approximiert. Dabei wird jedoch außer Acht gelassen, dass sich die absolute Höhe der Vorstandsvergütung nicht über verschiedene Unternehmen hinweg vergleichen lässt. Aus diesem Grund ziehen wir nicht die gesamte Vorstandsvergütung als Surrogat der Überwachungseffektivität 
heran, sondern untersuchen den Effekt auf den übermäßigen Anteil der Vergütung. Die übermäßige Vergütung bildet jenen Anteil der Vorstandsvergütung ab, welcher nicht auf unternehmens- und marktspezifische Determinanten zurückgeführt werden kann (Carter et al. 2016). Die Höhe der übermäßigen Vergütung spiegelt folglich den Umfang der Ineffizienz der Überwachung durch den Aufsichtsrat wider, der den Agency-Kosten des opportunistischen Verhaltens der Vorstände entspricht (Boyd 1994; Coles et al. 2014). Dies bedeutet, dass sich eine überhöhte Vergütung auf eine geringe Kontrolleffektivität des Aufsichtsrats zurückführen lässt (Winkler und Behrmann 2019).

Im Anschluss an die deskriptive Beurteilung der Frauenpräsenz im Aufsichtsrat bewerten wir die Überwachungseffektivität in Abhängigkeit der Gender-Diversity empirisch. Auf Basis der dargelegten Theorien argumentieren wir, dass Frauen im Aufsichtsrat im Zusammenhang mit einer verbesserten Überwachungseffektivität des Gremiums stehen. Dies wird darauf zurückgeführt, dass (Gender-) Diversity die Heterogenität im Kontrollgremium steigert und damit neue Denkweisen hervorruft, die sich wiederum positiv auf die Arbeit der Kontrolleure auswirken (Carter et al. 2003; Hillman und Dalziel 2003; Cumming et al. 2015). Darüber hinaus sind weibliche Aufsichtsräte in der Regel weniger vernetzt und seltener Mitglieder in einem Führungs- oder Kontrollorgan eines weiteren Unternehmens (Eulerich et al. 2014; Velte 2017b). Es liegt also die Vermutung nahe, dass sie unabhängiger als ihre männlichen Kollegen sind und insofern weniger Anreiz haben die Höhe der Vorstandsvergütung aus opportunistischen Gründen heraufzusetzen. Auch neigen Frauen dazu, mehr zu hinterfragen als Männer, was für die Überwachungseffektivität des Aufsichtsrats zuträglich sein kann (Aldamen et al. 2018). Darauf aufbauend leiten wir die folgende Hypothese $(\mathrm{H})$ ab:

H1a Es besteht ein positiver (negativer) Zusammenhang zwischen der Überwachungseffektivität (übermäßigen Vorstandsvergütung) und der Gender-Diversity der Aktionärsvertreter des Aufsichtsrats.

Auf der Grundlage der Critical Mass Theorie gehen wir darüber hinaus davon aus, dass der positive Einfluss weiblicher Aufsichtsräte mit ihrer Dominanz im Gremium zusammenhängt und sich diese erst ab einer bestimmten Anzahl behaupten können. Somit leiten wir die weitere Hypothese ab:

H1b Es besteht ein positiver (negativer) und nichtlinearer Zusammenhang zwischen der Überwachungseffektivität (übermäßigen Vorstandsvergütung) und der Gender-Diversity der Aktionärsvertreter des Aufsichtsrats.

Der Aufsichtsrat im deutschen Corporate Governance-System zeichnet sich, neben seiner Trennung vom Vorstand, durch die Mitbestimmung der Arbeitnehmer aus. Wie Duran und Pull (2014) präsentieren, finden sich Frauen häufiger innerhalb der Arbeitnehmer- als der Aktionärsvertreter im Aufsichtsrat wieder. Mithin ist der Einfluss weiblicher Arbeitnehmervertreterinnen im Aufsichtsrat in besonderer Weise zur berücksichtigen. Wir bewerten vor diesem Hintergrund zunächst, inwiefern die Arbeitnehmervertreter zur Geschlechterheterogenität des Aufsichtsrats beitra- 
gen. Darauf aufbauend prüfen wir anhand der folgenden Hypothesen, ob aufgrund der höheren Frauenpräsenz im Aufsichtsrat, durch Berücksichtigung der Arbeitnehmervertreter, eine verbesserte Überwachungseffektivität im Gremium vorherrscht:

H2a Es besteht ein positiver (negativer) Zusammenhang zwischen der Überwachungseffektivität (übermäßigen Vorstandsvergütung) und der Gender-Diversity des gesamten Aufsichtsrats (Aktionärs- und Arbeitnehmervertreter).

H2b Es besteht ein positiver (negativer) und nichtlinearer Zusammenhang zwischen der Überwachungseffektivität (übermäßigen Vorstandsvergütung) und der Gender-Diversity des gesamten Aufsichtsrats (Aktionärs- und Arbeitnehmervertreter).

H3a Es besteht ein positiver (negativer) Zusammenhang zwischen der Überwachungseffektivität (übermäßigen Vorstandsvergütung) und der Gender-Diversity der Arbeitnehmervertreter.

H3b Es besteht ein positiver (negativer) und nichtlinearer Zusammenhang zwischen der Überwachungseffektivität (übermäßigen Vorstandsvergütung) und der Gender-Diversity der Arbeitnehmervertreter.

Von einigen Kritikern der Geschlechterquote wird die These vertreten, dass diese dazu führt, dass wichtige Entscheidungen häufig in männerdominierte Ausschüsse delegiert werden, in welchen Aufsichtsrätinnen der Aktionäre und vor allem der Arbeitnehmer noch stärker unterrepräsentiert sind (Oehmichen et al. 2010; Bozhinov et al. 2017). Auch wird hinterfragt, inwiefern Arbeitnehmervertreter des Aufsichtsrats einen Einfluss auf strategische Entscheidungen, wie die Ausgestaltung der Vorstandsvergütung haben. Insbesondere, wenn die Aufsichtsräte der Anteilseigner im Vorstand eines anderen Unternehmens sitzen, haben diese ein geringeres Interesse daran, die Vorstandsgehälter gering zu halten, da dies das marktübliche Vergütungsniveau verringern würde. Die Arbeitnehmervertreter sollen hier eine regulierende Wirkung haben. Wird die Vorstandsvergütung jedoch im zuständigen Ausschuss ausgearbeitet, wird sie auch dem Einfluss des Gremiums entzogen (Raabe 2011). Die Frage, die hier zunächst deskriptiv zu prüfen wäre, ist, inwiefern die Wirkung der gesetzlichen Geschlechterquote umgangen wird, wenn zwar der Frauenanteil im Aufsichtsrat, jedoch nicht in den Ausschüssen steigt. Nachfolgend untersuchen wir empirisch die folgende Hypothese:

H4a Es besteht ein positiver (negativer) Zusammenhang zwischen der Überwachungseffektivität (übermäßigen Vorstandsvergütung) und der Gender-Diversity im Vergütungsausschuss des Aufsichtsrats.

H4b Es besteht ein positiver (negativer) und nichtlinearer Zusammenhang zwischen der Überwachungseffektivität (übermäßigen Vorstandsvergütung) und der Gender-Diversity im Vergütungsausschuss des Aufsichtsrats. 


\section{Untersuchungsdesign}

\subsection{Regressionsmodell}

Der empirische Zusammenhang zwischen Gender-Diversity (Diversity) im Aufsichtsrat und seiner Überwachungseffektivität wird anhand des folgenden Regressionsmodells getestet:

$$
\begin{aligned}
\text { ExcPay } / \text { vAR ExcPay }= & \beta_{0} * \text { Konstante }+\beta_{1} * \text { Diversity }+\beta_{2} * \text { ARGrösse } \\
& +\beta_{3} * \text { FreeFloat }+\beta_{4} * \text { Mandate }_{\mathrm{D}}+\beta_{5} * \text { Medien }_{\mathrm{LN}} \\
& +\beta_{6} * \text { AR Sitzungen }+\beta_{7} * \text { Verschuldung } \\
& +\beta_{8} * \text { Perioden }_{\mathrm{D}}+\beta_{9} * \text { Unternehmen }_{\mathrm{D}}+\mu
\end{aligned}
$$

Die Überwachungseffektivität wird dabei durch die (variable) übermäßige Vergütung (ExcPay bzw. varExcPay) approximiert, die anhand der nachfolgenden Regressionsgleichung ermittelt wird und dem Residuum $(\mu)$ der Schätzung entspricht:

$$
\begin{aligned}
\mathrm{VV}_{\mathrm{LN}} / \mathrm{VAR}_{\mathrm{VN}}= & \beta_{0} * \text { Konstante }+\beta_{1} * \text { MWBW }+\beta_{2} * \text { ROA } \\
& +\beta_{3} * \text { TotalAssets }_{\mathrm{LN}}+\beta_{4} * \text { TotalReturn }_{\mathrm{LN}}+\beta_{5} * \mathrm{DAX}_{\mathrm{D}} \\
& +\beta_{6} * \text { Branchen }_{\mathrm{D}}+\beta_{7} * \text { Perioden }_{\mathrm{D}}+\beta_{8} * \text { Unternehmen }_{\mathrm{D}} \\
& +\mu
\end{aligned}
$$

\subsection{Abhängige Variable: Überwachungseffektivität}

Die (variable) übermäßige Vorstandsvergütung (ExcPay bzw. vaRExcPay) dient als abhängige Variable der empirischen Untersuchung und als Proxy der Überwachungseffektivität. Die übermäßige Vergütung bildet den Anteil der Vergütung ab, welcher sich nicht durch markt- und unternehmensspezifische Determinanten erklären lässt. Da die absolute Vorstandsvergütung bspw. von der Größe des Unternehmens, dem Unternehmenserfolg und der Branche abhängt, stellt sie keine geeignete Kennzahl für die Überwachungseffektivität dar (Rapp und Wolff 2010). Die übermäßige Vergütung berücksichtigt dagegen die Angemessenheit, Üblichkeit und Nachhaltigkeit der Vergütung und bildet lediglich den ineffizienten Anteil der Vergütung ab (Winkler und Behrmann 2019). Die Variablen ExcPay und ${ }_{V A R}$ ExcPay ermitteln wir im Residuum der Regression des Unternehmenserfolges ( $M W B W=$ Marktwert-Buchwert-Verhältnis, ROA, TotalReturn ${ }_{L N}$ ), der Unternehmensgröße (TotalAssets ${ }_{L N}$ ) sowie von Index-, Branchen-, Perioden- und Unternehmenseffekten $\left(D A X_{D}\right.$, Branchen $n_{D}$, Perioden $_{D}$, Unternehmen ${ }_{D}$ ) auf die logarithmierte gesamte bzw. variable pro-KopfDurchschnittsvergütung des Vorstands (Winkler und Behrmann 2019). Die gesamte Vorstandsvergütung $\left(V V_{L N}\right)$ entspricht dabei der durchschnittlich zugeflossenen Gesamtvergütung (Festvergütung, Nebenleistungen, Versorgungsaufwand und Performance-Bonus) je Vorstandsmitglied und die performanceabhängige Vergütung $\left({ }_{\text {VAR }} V V_{L N}\right)$ der Differenz aus Gesamt- und Festvergütung dividiert durch die Anzahl 
der Vorstandsmitglieder. Um Größenunterschiede der Gehälter zwischen den Unternehmen zu verdichten, verwenden wir den natürlichen Logarithmus der (variablen) Vergütung (Usman et al. 2018).

$$
\begin{aligned}
& \mathrm{LN}(\text { Vorstandsvergütung })=\frac{\sum \text { zugeflossene Gesamtvergütung }}{\text { Anzahl der Vorstandsmitglieder }}=\mathrm{VV}_{\mathrm{LN}} \\
& \text { LN(variable Vorstandsvergütung) } \\
& =\frac{\sum \text { zugeflossene Gesamtvergütung }-\sum \text { Festvergütung }}{\text { Anzahl der Vorstandsmitglieder }}={ }_{\mathrm{VAR}} \mathrm{VV}_{\mathrm{LN}}
\end{aligned}
$$

\subsection{Unabhängige Variable: Gender-Diversity}

In der bisher veröffentlichten Forschung wurde eine Reihe unterschiedlicher Heterogenitätsmaße verwendet. Diese führten zum Teil zu unterschiedlichen Ergebnissen und einer geringen Vergleichbarkeit der verschiedenen Studien (Harrison und Klein 2007; Boerner et al. 2012). Aus diesem Grund und, um die Repräsentanz weiblicher Aufsichtsräte umfangreich zu erfassen, berechnen wir mehrere Diversity-Kennzahlen (Diversity), welche sich in der Corporate Governance-Forschung etabliert haben. Wir berechnen die Kennzahlen jeweils für die Aktionärsvertreter des Aufsichtsrats, den gesamten Aufsichtsrat, sowie für den für die Vorstandsvergütung zuständigen Ausschuss (i.d.R. Präsidial-, Personal- oder Vergütungsausschuss). Für den ersten Abschnitt der Untersuchung erfassen wir erste unabhängige Variable der Frauenanteil (Frauenanteil) je Aufsichtsrat. Zudem wird anhand des Blau- und ShannonIndex die Geschlechterheterogenität im Kontrollgremium ermittelt. Die Heterogenitätsmaße nehmen Werte zwischen 1 (= ausgeglichen) und 0 (= unausgeglichen) an und werden wie folgt berechnet (Harrison und Klein 2007):

$$
\begin{aligned}
& \text { Blau }=1-\sum_{\mathrm{m}=1}^{\mathrm{k}} \mathrm{a}_{\mathrm{m}}^{2} \\
& \text { Shannon }=-\sum_{\mathrm{m}=1}^{\mathrm{k}} \mathrm{a}_{\mathrm{m}} \ln \mathrm{a}_{\mathrm{m}}
\end{aligned}
$$

mit:

$\mathrm{k}=$ Anzahl der Kategorien (=2)

$\mathrm{a}_{\mathrm{m}}=$ Anteil der Aufsichtsratsmitglieder mit Merkmal m (= weiblich/männlich)

Des Weiteren werden zwei Dummy-Variablen berücksichtigt, die angeben, ob mindestens eine Frau $\left(\right.$ Frauen $\left._{D}\right)$ bzw. mindestens $30 \%$ weibliche Mitglieder $\left(\right.$ Frauen $\left._{30}\right)$ im Gremium vertreten sind. Letztere Variable drückt aus, inwiefern das Unternehmen die gesetzliche Geschlechterquote bereits erreicht hat.

Im zweiten Abschnitt wird durch weitere Regressionen getestet, inwiefern der Zusammenhang variiert, wenn mehr oder weniger Frauen im Aufsichtsrat vertreten sind. Dazu werden vier Dummy-Variablen in das Regressionsmodell (Aktionärsver- 
treter, Arbeitnehmervertreter und gesamter Aufsichtsrat) integriert, die angeben, ob keine Frau $\left(\right.$ Frauen $_{0}=$ Referenzkategorie), genau eine Frau $\left(\right.$ Frauen $\left._{1}\right)$, zwei Frauen $\left(\right.$ Frauen $\left._{2}\right)$, drei Frauen $\left(\right.$ Frauen $\left._{3}\right)$ oder mindestens vier Frauen $\left(\right.$ Frauen $\left._{4}\right)$ dem Gremium angehören. Da keinem Vergütungsausschuss mehr als drei Frauen angehören, verwenden wir in der entsprechenden Regressionsgleichung lediglich drei DummyVariablen.

\subsection{Kontrollvariablen}

Als Kontrollvariablen werden vor allem aufsichtsratsspezifische Kennzahlen verwendet. Die unternehmens- und marktspezifischen Parameter, die bereits im Modell zur Schätzung der übermäßigen Vergütung und damit in der abhängigen Variablen der folgenden Regression enthalten sind, werden nicht noch einmal im Grundmodell berücksichtigt. Zunächst wird dazu die Anzahl der Mitglieder je Aufsichtsrat durch die Variable ARGrösse erfasst. Der Umfang der Aufsichtsratstätigkeit wird in der Variable ARSitzungen approximiert, indem sie die Anzahl der Aufsichtsratssitzungen im Geschäftsjahr zählt. Die Dummy-Variable Mandate $_{D}$, die angibt, ob mindestens 30\% der Mitglieder des Gremiums drei oder mehr Mandate in weiteren Kontroll- und Führungsorganen halten, spiegelt die Erfahrung bzw. Ablenkung des Gremiums quantitativ wider. Inwiefern Mehrfachmandate vor- bzw. nachteilig für die Arbeit des Aufsichtsrats sind, ist in der Forschung nicht abschließend geklärt. Auf der einen Seite wird argumentiert, dass Aufsichtsräte mit vielen Mandaten zu abgelenkt sind, ihrer Kontrollaufgabe gewissenhaft nachzugehen. Auf der anderen Seite wird davon ausgegangen, dass nur Aufsichtsräten mit hoher Expertise mehrere Mandate angeboten werden und diese eine höhere Überwachungseffektivität aufweisen (Arnegger und Hoffmann 2014; Handschumacher et al. 2019). Darüber hinaus wird anhand der Variablen FreeFloat und Verschuldung der Einfluss der Eigentümerstruktur und externer Investoren kontrolliert. Dabei gibt FreeFloat den Anteil der im Streubesitz befindlichen Aktien und Verschuldung den Verschuldungsgrad des Unternehmens an. Auf Basis der Überlegungen von Rapp et al. (2010) sowie Handschumacher et al. (2019), dass Corporate Governance-Entscheidungen durch die Medienpräsenz des Unternehmens beeinflusst werden können, wird die Variable Medien.TA in die Regressionsgleichung integriert. Die Variable ergibt sich aus der Trefferanzahl des Unternehmens in der Suchmaschine Google News. Da wir unterstellen, dass die Medienpräsenz abhängig von der Größe des Unternehmens ist, setzten wir die Trefferanzahl ins Verhältnis zur Unternehmensgröße, gemessen an der logarithmierten Bilanzsumme (TotalAsset $s_{L N}$ ). In allen Regressionen werden Perioden- und Unternehmenseffekte kontrolliert. Schließlich inkludieren wir die Kontrollvariable Frauenanteil.Vorstand, die den Anteil weiblicher Mitglieder im Vorstand angibt. Auf der Grundlage empirischer Evidenzen gehen wir davon aus, dass Frauen seltener und weniger intensiv über ihre Vergütung verhandeln und nach einer abnormalen Vergütung streben, was dazu führt, dass ExcPay bei einem hohen Frauenanteil im Gremium geringer ausfallen würde (Babcock und Laschever 2003; Perryman et al. 2016). 


\subsection{Stichprobe und Datengrundlage}

Die empirische Untersuchung basiert auf einer Grundgesamtheit von 160 deutschen Unternehmen, die über den Zeitraum von 2015 bis 2017 in den größten deutschen Indizes DAX30, MDAX, SDAX und TecDAX gelistet waren. Die Grundgesamtheit wurde um Banken, Versicherungen und Finanzdienstleister sowie Unternehmen mit ausländischer ISIN reduziert, womit sich die Untersuchung auf eine Stichprobe von 132 Unternehmen und 396 Beobachtungen stützt. Als Datenquelle dienen insbesondere die Geschäftsberichte der Unternehmen (2015-2017) sowie die Datenbank Thomson Reuters Datastream. Aus den Geschäftsberichten wurden händisch alle Aktionärs- und Arbeitnehmervertreter des Aufsichtsrats entnommen, sowie je Aufsichtsratsmitglied das Geschlecht und für die Aktionärsvertreter die Anzahl an Mandaten in weiteren nationalen und internationalen Kontroll- und Führungsorganen. Zudem erhoben wir die Mitglieder des für Vorstandsvergütung zuständigen Aufsichtsratsausschuss (i.d.R. Präsidial- bzw. Personalausschusses). Je Aufsichtsrat haben wir zudem die Anzahl der Sitzungen und die Größe des Gremiums erfasst. Als Datenquelle der Vorstandsvergütung und -zusammensetzung dienten ebenfalls die Geschäftsberichte. Die Informationen zur Unternehmensperformance, -größe und -verschuldung sowie zum Streubesitz sind der Datenbank Thomson Reuters Datastream entnommen. Schließlich wurde die Medienpräsenz anhand der Trefferzahl der Unternehmen für den Zeitraum vom 01.01.2015 bis zum 31.12.2017 in der Suchmaschine Google News erhoben.

\subsection{Methode}

Zur empirischen Untersuchung des Zusammenhangs von Gender-Diversity im Aufsichtsrat und seiner Überwachungseffektivität führen wir mehrere Panelregressionsanalysen durch. Die Regressionen werden mit fixen Unternehmenseffekten (FixedEffects-Modell) geschätzt, um nicht beobachtbare Unternehmenseigenschaften zu kontrollieren. Anhand des panelrobusten Hausman-Tests beurteilen wir je Modellspezifikation, inwiefern sich darüber hinaus, aus formellen Gesichtspunkten, eine Schätzung mit zufälligen Unternehmenseffekten (Random-Effects-Modell) anbietet. Die Nullhypothese des Hausman-Tests, die besagt, dass das unternehmensspezifische Residuum signifikant mit der unabhängigen Variable korreliert, wird verworfen, wenn sich der Fixed-Effects- und der Random-Effects-Schätzer signifikant voneinander unterscheiden ( $\left.\mathrm{prob}>\mathrm{chi}^{2}<0,1\right)$ (Hausman 1978). Basierend auf den überwiegend signifikanten Ergebnissen des Hausman-Tests und einer inhaltlichen Präferenz für Fixed-Effects-Modelle, werden die Regressionsanalysen mit fixen Unternehmenseffekten durchgeführt. Die einheitliche Durchführung aller Regressionsanalysen mit fixen Unternehmenseffekten (auch bei abweichender Empfehlung durch den Hausman-Test) hat den Vorteil, dass eine Vergleichbarkeit der Ergebnisse sichergestellt werden kann. Ist dies der Fall, empfiehlt der Hausmann-Test Random-EffectsModelle zu verwenden. Unser Regressionsmodell basiert zudem auf der Annahme, dass weder Multikollinearität noch Heteroskedastizität vorliegt. Um Multikollinearität auszuschließen, werden die paarweisen Pearson-Korrelationskoeffizienten ermittelt. Liegen die Koeffizienten unterhalb des kritischen Werts von 0,8 ist nicht von 
Multikollinearität auszugehen (Gujarati und Porter 2010). Zudem wird je Regressionsgleichung der Varianzinflationsfaktor (VIF) berechnet. Bei möglichst niedrigen VIFs kann Multikollinearität weitestgehend ausgeschlossen werden, wobei ein Wert von maximal 10,0 angestrebt wird (Schneider 2009). In den nachfolgenden Regressionen wird ein VIF von 2,0 nicht überschritten, womit nicht von Multikollinearität auszugehen ist. Zur Vermeidung von Verzerrungen durch Heteroskedastizität werden alle Regressionsanalysen mit robusten Standardfehlern geschätzt. Des Weiteren kann Endogenität in den Variablen die Schätzergebnisse verzerren. Wenn die unabhängige Variable (Diversity) mit dem Residuum korreliert [E $(\mu \mathrm{IX})=0]$ führt dies dazu, dass der Wirkungszusammenhang der abhängigen und unabhängigen Variablen nicht eindeutig identifiziert werden kann (Börsch-Supan und Köke 2002; Proppe 2009). In einigen empirischen Untersuchungen zur (übermäßigen) Vorstandsvergütung wird unterstellt, dass Endogenitätsprobleme weiterstgehend ausgeschlossen werden können, da sich diese im Zusammenhang mit der (übermäßigen) Vorstandsvergütung bereits auf inhaltlicher Ebene verwerfen lassen (Winkler und Behrmann 2019; Handschumacher et al. 2019). Darüber hinaus können fixe Unternehmenseffekte, die wir auch in unserer Untersuchung verwenden, Endogenitätsprobleme reduzieren (Adams und Ferreira 2009). Es besteht jedoch nach wie vor die Möglichkeit, dass Diversity keine Zufallsvariable, sondern endogen gegeben ist. Dies bedeutet, dass Frauen aufgrund bestimmter Charakteristika des Unternehmens in dessen Aufsichtsrat berufen werden. Wie zuvor erläutert, gehen wir davon aus, dass weibliche Vorstände seltener opportunistische Verhaltensweisen hinsichtlich des Strebens nach einer übermäßigen Vergütung an den Tag legen. Des Weiteren zeigen empirische Evidenzen, dass Frauen häufiger in Gremium berufen werden, wenn bereits eine weibliche Managerin bzw. Kontrolleurin vertreten ist (Oehmichen et al. 2010; Hutchinson et al. 2015). Die abhängige und die unabhängige Variable der Untersuchung, das heißt sowohl die übermäßige Vorstandsvergütung als auch das Ausmaß der Gender-Diversity im Aufsichtsrat können beispielsweise von der Existenz weiblicher Vorstände abhängen. Wir berücksichtigen diese Effekte weitestgehend durch Kontrollvariablen, trotzdem können nicht-beobachtbare Unternehmenscharakteristika nie ausgeschlossen werden und dazu führen, dass wir einen Zusammenhang aufdecken, dem keine Kausalität zugrunde liegt (Sila et al. 2016). In der Forschung wird diesem Problem teilweise durch zweistufige Schätzmodelle (2-stage-least-square) begegnet. Dabei kommen Instrumental-Variablen zum Einsatz, die mit der Gender-Diversity im Aufsichtsrat hoch korreliert sind und nicht im Zusammenhang mit der Vorstandsvergütung stehen. Kritiker der Methode bringen jedoch an, dass keine geeigneten InstrumentalVariablen existieren, die diese Kriterien erfüllen und diese zu Verzerrungen der Schätzergebnisse führen können (Ittonen et al. 2010). Aus diesem Grund sehen wir von der Verwendung dieser Methode ab. 


\section{Empirische Analyse}

\subsection{Deskriptive Statistik}

Unser Datensatz umfasst für das Jahr 2017 insgesamt 1470 (2016: 1450; 2015: 1450) Aufsichtsratsmitglieder. Bei 132 Unternehmen liegt die durchschnittliche Anzahl somit bei 11,1 (2016: 11,0; 2015: 11,0) Aufsichtsratsmitgliedern. Von diesen Aufsichtsräten sind 1081 (2016: 1088; 2015: 1145) männlich und 389 (2016: 362; 2015: 305) weiblich. Dies entspricht einer durchschnittlichen Anzahl von 3,0 (2016: 2,7; 2015: 2,3) Aufsichtsrätinnen bzw. einem Frauenanteil von 23,3\% (2016: 22,2 \%; 2015: 18,7\%) im Kontrollorgan. Der Frauenanteil stieg somit von 2015 bis 2017 um 4,6 Prozentpunkte. Damit werden im Jahr 2017 bereits 41,6\% (2016: 36,4\%; 2015: $22,7 \%$ ) der Unternehmen der gesetzlichen Geschlechterquote von $30 \%$ gerecht. Dagegen existiert im dreijährigen Betrachtungszeitraum kein Unternehmen, in dem mehr Frauen als Männer im Kontrollgremium vertreten sind, während 14,4\% der Gremien (2016: 15,2\%; 2015: 19,7\%) durch ausschließlich männliche Aufsichtsräte kontrolliert werden. Auch in Hinblick auf die Vorsitzposition sind weibliche Vertreter stark unterrepräsentiert. Lediglich in vier bzw. 3,0\% (2016 und 2015: vier bzw. 3,0\%) der betrachteten Unternehmen wird der Aufsichtsrat durch eine Vorsitzende vertreten. Abb. 1 verdeutlicht den Zuwachs weiblicher Aufsichtsräte in den letzten Jahren. Darüber hinaus verdeutlich die Abbildung, dass der Frauenanteil im Aufsichtsrat insbesondere durch Arbeitnehmerinnen determiniert wird. Hier liegt der durchschnittliche Anteil 2017 bei 27,2\% (2016: 24,9; 2015:21,3\%), wohingegen der Anteil weiblicher Aktionärsvertreter 24,5\% (2016: 22,1\%; 2015: 18,6\%) entspricht. Es wird des Weiteren deutlich, dass Frauen insbesondere im Vergütungsausschuss stark unterrepräsentiert sind. Im Mittel liegt der Frauenanteil hier bei 12,4\% (2016: $12,7 \% ; 2015: 13,1 \%$ ) und ist damit in den letzten Jahren sogar weitergesunken.

Ferner zeigt die deskriptive Auswertung, dass der Anteil an Aufsichtsrätinnen in Unternehmen des DAX (2017: 30,9\%; 2016: 28,5\%; 2015: 24,6\%) im Mittel höher ist als in Unternehmen des SDAX (2017: 23,4\%; 2016: 23,1\%; 2015: 19,8). Die deskriptive Statistik der abhängigen, unabhängigen und Kontrollvariablen ist der Tab. 1 zu entnehmen.

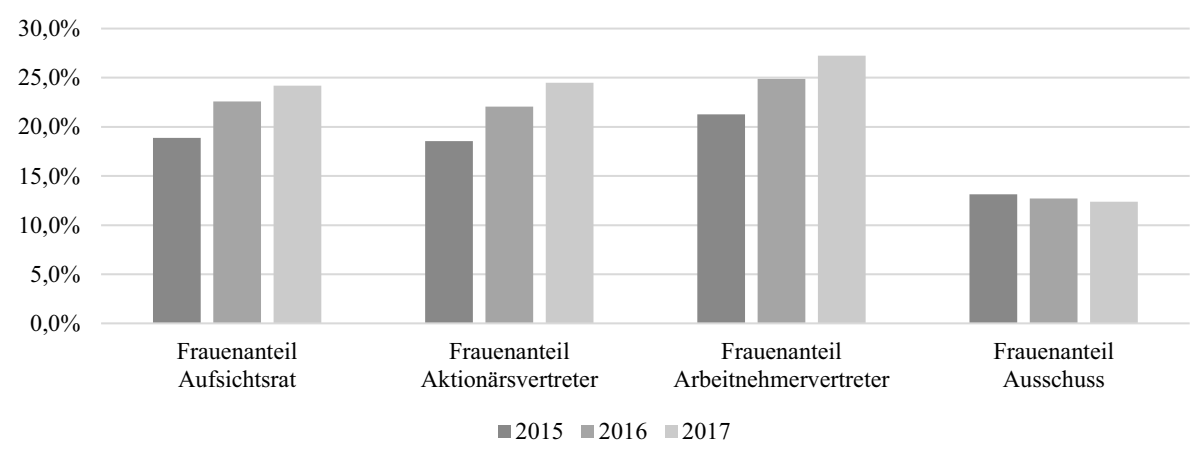

Abb. 1 Anteil weiblicher Aufsichtsräte von 2015 bis 2017 
Tab. 1 Deskriptive Statistik

\begin{tabular}{|c|c|c|c|c|c|}
\hline Variablen & $N$ & Mittelwert & Standardabweichung & Min & Max \\
\hline \multicolumn{6}{|l|}{ Abhängige Variablen } \\
\hline ExcPay & 371 & 0,000 & 0,482 & $-2,290$ & 2,267 \\
\hline VARExcPay & 365 & 0,000 & 0,709 & $-2,534$ & 2,251 \\
\hline \multicolumn{6}{|l|}{ Unabhängige Variablen } \\
\hline Frauenanteil.Gesamt & 395 & 0,219 & 0,123 & 0,000 & 0,500 \\
\hline Frauenanteil.Aktionär & 395 & 0,217 & 0,139 & 0,000 & 0,666 \\
\hline Frauenanteil.Ausschuss & 342 & 0,124 & 0,177 & 0,000 & 0,750 \\
\hline Frauenanteil.Arbeitnehmer & 268 & 0,240 & 0,174 & 0,000 & 1,000 \\
\hline Blau.Gesamt & 395 & 0,311 & 0,152 & 0,000 & 0,500 \\
\hline Shannon.Gesamt & 395 & 0,466 & 0,214 & 0,000 & 0,693 \\
\hline Frauen $_{D}$.Gesamt & 395 & 0,856 & 0,352 & 0,000 & 1,000 \\
\hline Frauen $_{30}$. Gesamt & 395 & 0,347 & 0,477 & 0,000 & 1,000 \\
\hline \multicolumn{6}{|l|}{ Kontrollvariablen } \\
\hline ROA & 395 & 5,999 & 5,485 & $-15,800$ & 28,280 \\
\hline TotalReturn $_{\mathrm{LN}}$ & 395 & 6,451 & 1,554 & 1,638 & 10,129 \\
\hline MWBW & 395 & 3,257 & 2,873 & $-12,710$ & 18,960 \\
\hline ARGrösse.Gesamt & 395 & 11,147 & 5,170 & 2,000 & 21,000 \\
\hline ARGrösse.Aktionär & 395 & 6,722 & 2,193 & 2,000 & 12,000 \\
\hline ARGrösse.Ausschuss & 342 & 3,792 & 1,666 & 0,000 & 8,000 \\
\hline ARSitzungen & 392 & 6,525 & 2,914 & 0,000 & 26,000 \\
\hline Mandate $_{\mathrm{D}}$ & 395 & 0,364 & 0,235 & 0,000 & 1,000 \\
\hline Verschuldung & 395 & 33,641 & 22,110 & 0,000 & 91,700 \\
\hline FreeFloat & 395 & 66,752 & 23,662 & 14,000 & 100,000 \\
\hline TotalAssetsLN & 395 & 15,138 & 1,706 & 11,180 & 19,837 \\
\hline Medien $_{\text {.TA }}$ & 395 & 50,727 & 244,333 & 0,000 & 2897,471 \\
\hline Frauenanteil.Vorstand & 391 & 0,060 & 0,153 & 0,000 & 2,000 \\
\hline
\end{tabular}

Die Tabelle präsentiert die deskriptive Statistik der herangezogenen Variablen. Als Datengrundlage fungiert der Paneldatensatz mit 396 Unternehmensjahren. Aufgrund fehlender werte reduziert sich die Anzahl der Beobachtungen der übermäßigen Vorstandsvergütung (ExcPay/VARExcPay) auf 371 bzw. 365

\subsection{Korrelationsanalyse}

Die Tab. 2 bildet die Ergebnisse der Korrelationsanalyse ab. Die Analyse verdeutlicht, dass die Diversity-Kennzahlen, ausschließlich der Variablen Frauen ${ }_{30}$.Gesamt und Frauenanteil.Aktionär, in einem positiven und signifikanten Verhältnis zur (variablen) übermäßigen Vergütung stehen. Da lediglich die Korrelationskoeffizienten der Vergütungskennzahlen ExcPay und ${ }_{V A R}$ ExcPay sowie der Variablen zur Messung der Gender-Diversity einen kritischen Wert von 0,8 überschreiten bzw. sich diesem annähern und diese nicht im gleichen Regressionsmodell Verwendung finden, ist nicht von Multikollinearität auszugehen (Gujarati und Porter 2010). 


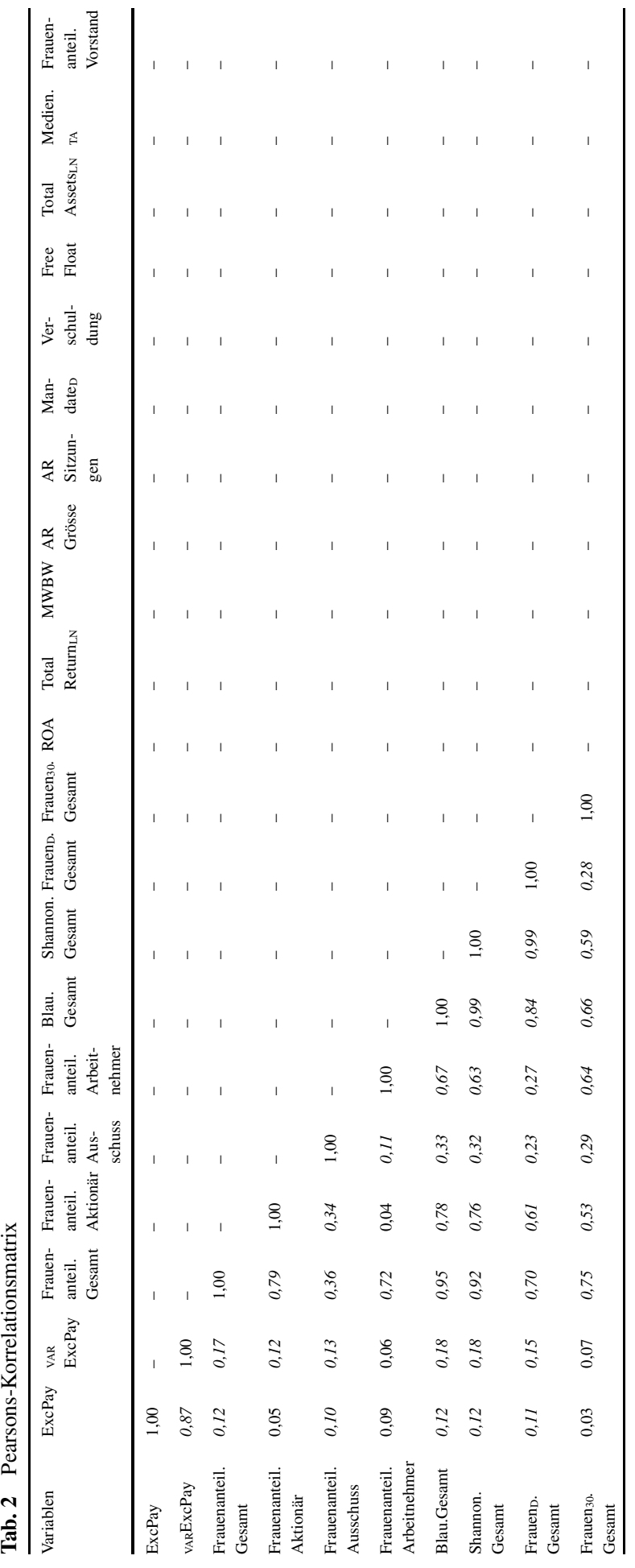




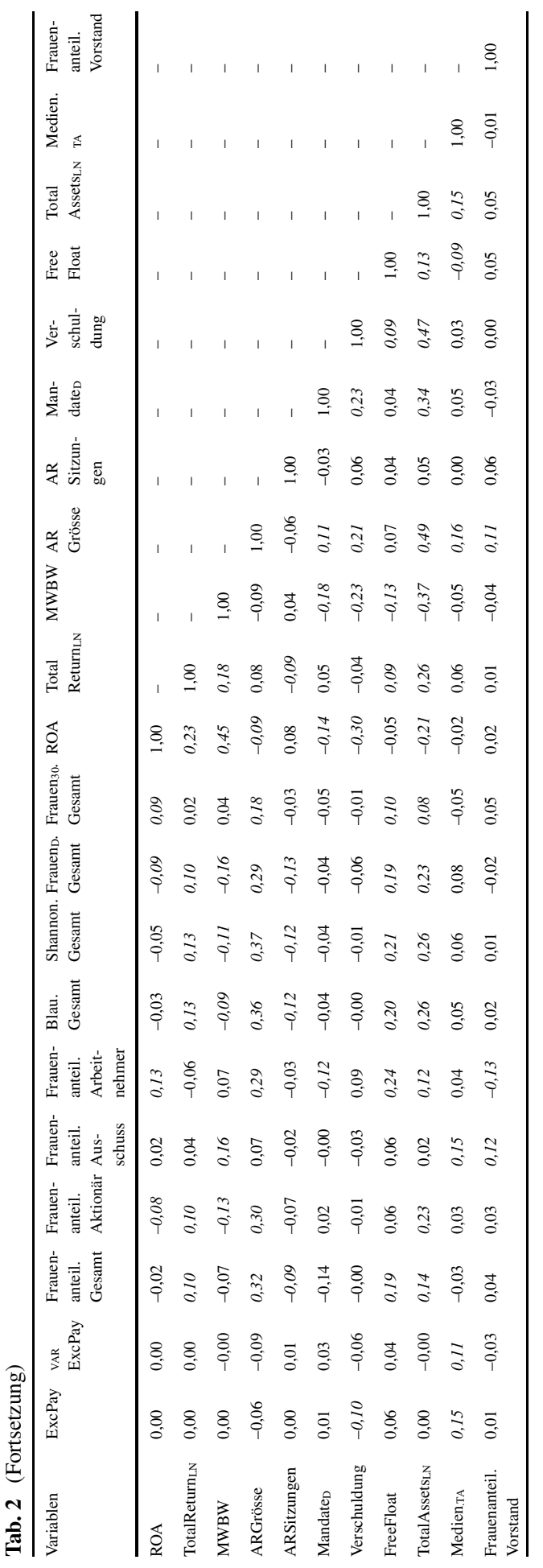




\subsection{Regressionsanalyse}

Zur Untersuchung der prognostizierten Zusammenhänge führen wir mehrere Regressionsanalysen mit fixen Unternehmenseffekten durch.

\subsubsection{Linearer Zusammenhang zwischen Gender-Diversity und Überwachungseffektivität}

Die Ergebnisse der empirischen Untersuchung zum Zusammenhang zwischen der Gender-Diversity im Aufsichtsrat und der übermäßigen Vorstandsvergütung als Proxy für die Überwachungseffektivität des Kontrollgremiums sind in den Tab. 3, 4, 5 und 6 dargestellt. Die Assoziation schätzen wir anhand der gesamten übermäßigen Vorstandsvergütung (ExcPay) bzw. der variablen übermäßigen Vorstandsvergütung (vaRExcPay) und den jeweils fünf unabhängigen Diversity-Variablen des gesamten Aufsichtsrats (Tab. 3), der Aktionärsvertreter (Tab. 4), der Arbeitnehmervertreter (Tab. 5), sowie des Vergütungsausschusses (Tab. 6). Die Regressionen eröffnen dabei konsistente Ergebnisse, die eine negative Assoziation zwischen der Repräsentanz weiblicher Aufsichtsräte und der (variablen) übermäßigen Vergütung identifizieren.

Die Regressionen der Diversity-Kennzahlen des gesamten Aufsichtsrats auf die

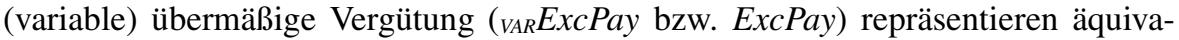
lente Ergebnisse zur vorstehenden Untersuchung (Tab. 3). Die $\beta$-Koeffizienten der unabhängigen Diversity-Variablen liegen dabei zwischen $-1,395$ und $-0,171$ bzw. $-0,564$ und $-0,110$. Die Regressionen auf ${ }_{V A R} E x c P a y$ ist durchgehen signifikant. Dagegen liefert die Schätzung der abhängigen Variable ExcPay durch die unabhängigen Variablen Frauenanteil und Frauen $_{30}$ keine signifikanten Resultate. Nichts desto trotz weisen die überwiegend signifikanten Ergebnisse auf einen negativen $\mathrm{Zu}$ sammenhang zwischen der Gender-Diversity im Aufsichtsrat und der übermäßigen Vergütung hin. Die Befunde konstatieren damit die vorteilhafte Wirkung von Frauen im Überwachungsausschuss und bekräftigen die Hypothese 2a.

Die Regressionen der Diversity-Kennzahlen der Aktionärsvertreter auf die (variable) übermäßige Vergütung (vaRExcPay bzw. ExcPay) weisen für die unabhängigen Variablen $\beta$-Koeffizienten zwischen $-1,187$ und $-0,137$ bzw. $-0,581$ und $-0,070$ aus, die, ausschließlich der Variablen Frauen 30 , signifikant sind (Tab. 4). Insgesamt deuten die Ergebnisse darauf hin, dass der nicht ökonomisch determinierte Anteil der Vorstandsvergütung, bei steigender Gender-Diversity sinkt. Dies suggeriert, dass ein genderheterogener Aufsichtsrat den Vorstand effektiver überwacht, und dies mit einer verbesserten Corporate Governance des Unternehmens einhergeht. Die vorstehenden Ergebnisse stehen im Einklang mit den Studien von Adams und Ferreira (2009), Lucas-Pérez et al. (2015) sowie Baixauli-Soler et al. (2016), die ebenfalls einen vorteilhaften Effekt von Frauen auf die Überwachungseffektivität postulieren. Die Befunde der Untersuchung stützen damit die Hypothese 1a.

Nach der Betrachtung des gesamten Aufsichtsrats sowie der Aktionärsvertreter vervollständigt folgend die Analyse zur Wirkung der Gender-Diversity der Arbeitsnehmervertreter das Bild. Die Regressionen der Diversity-Kennzahlen der Arbeitnehmervertreter auf die (variable) übermäßige Vergütung (vaRExcPay bzw. ExcPay) 


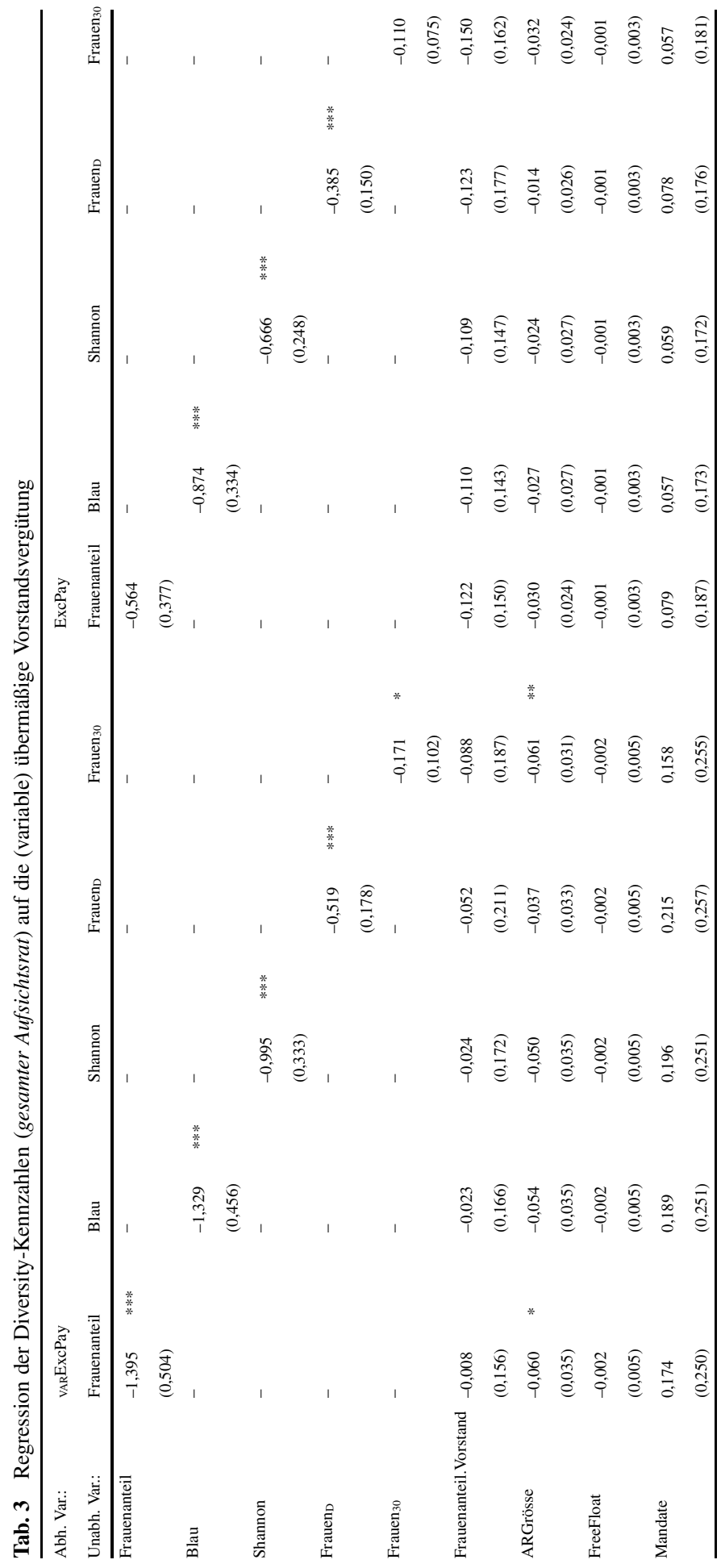




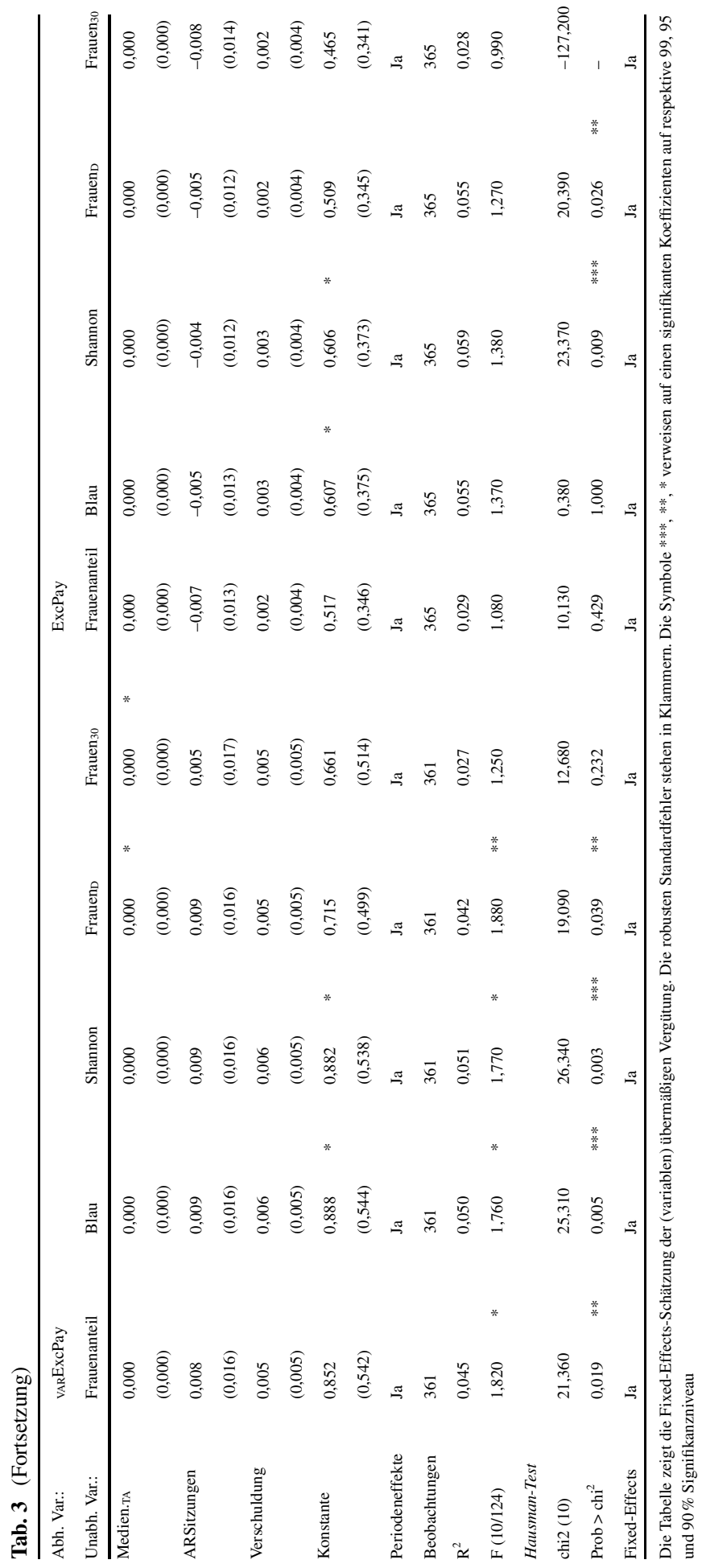




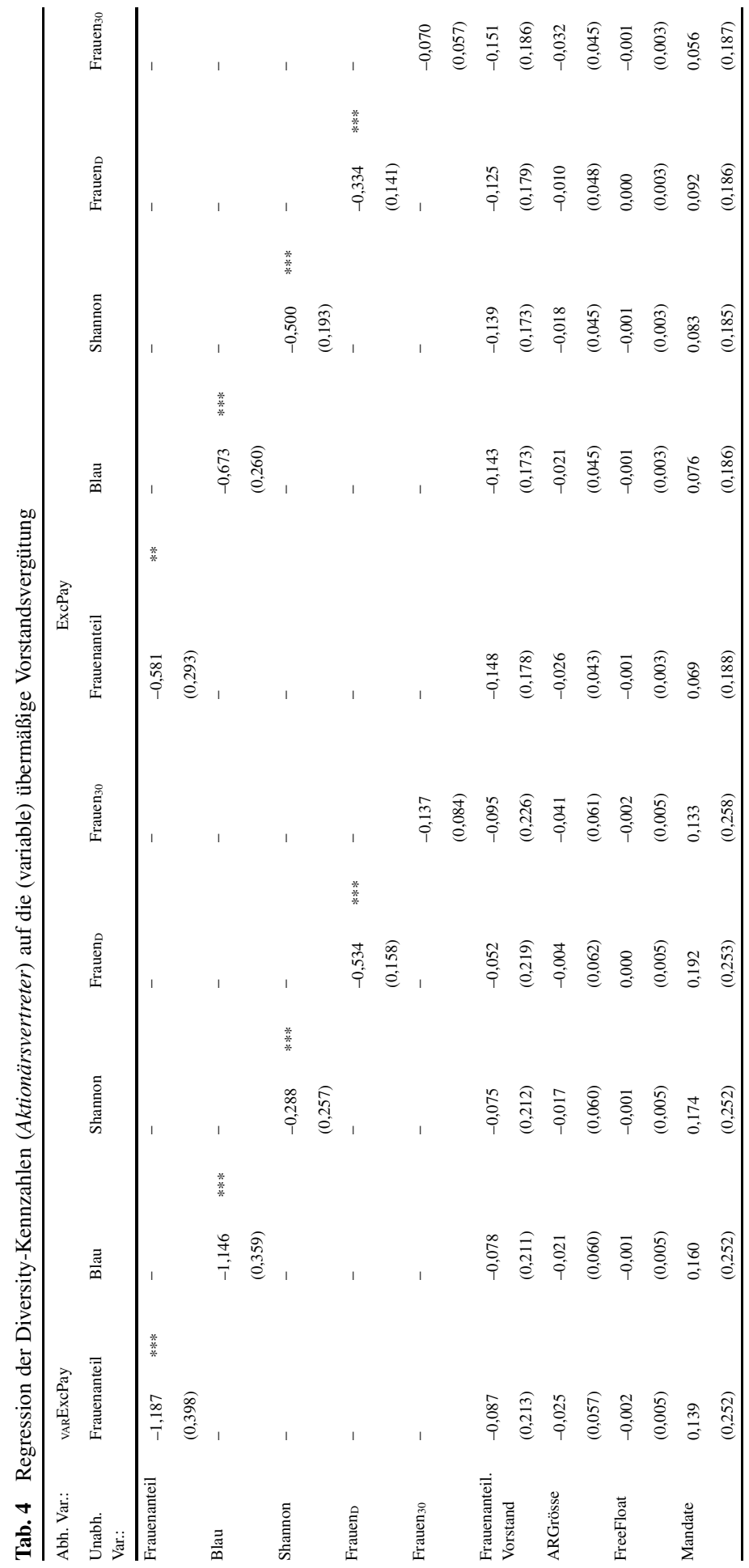




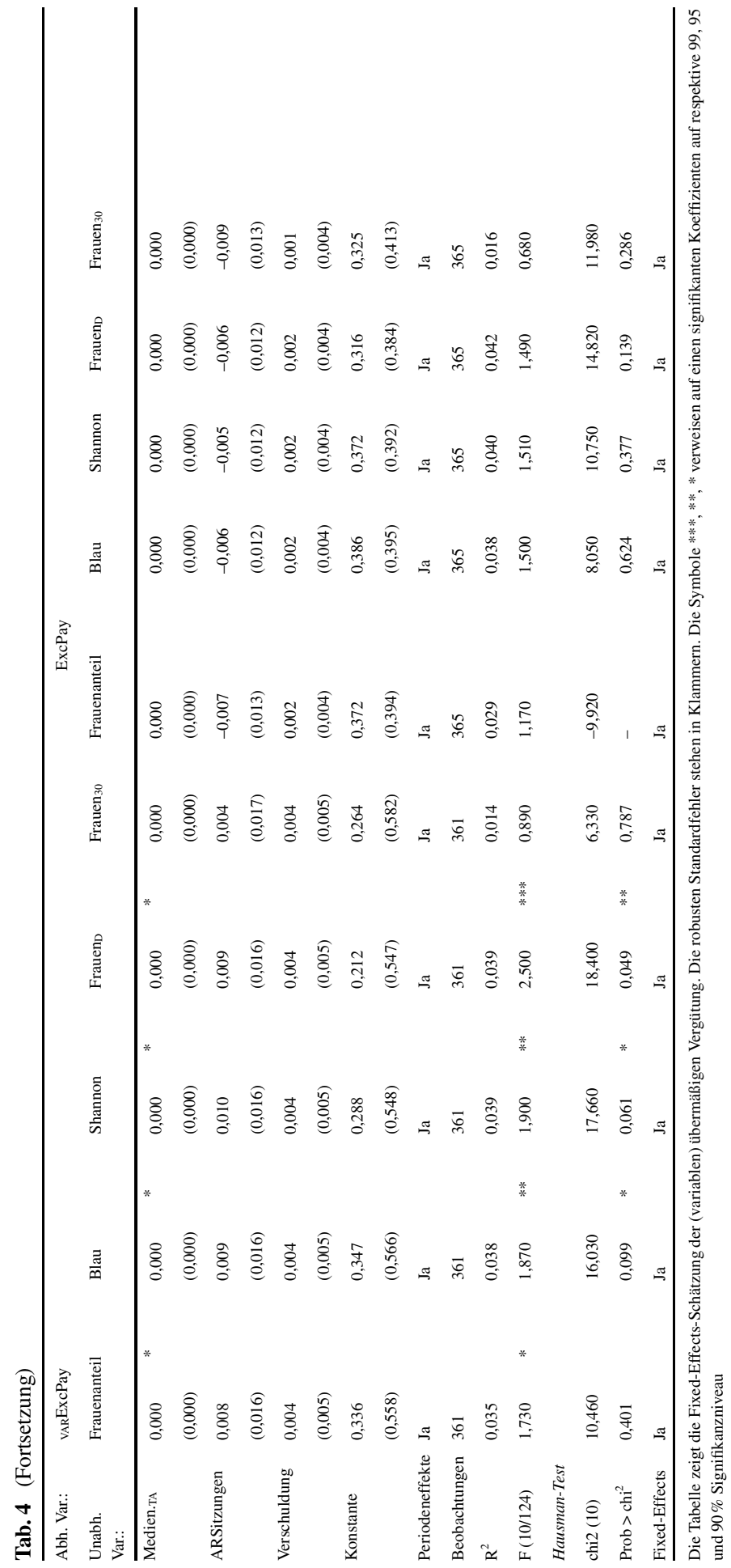




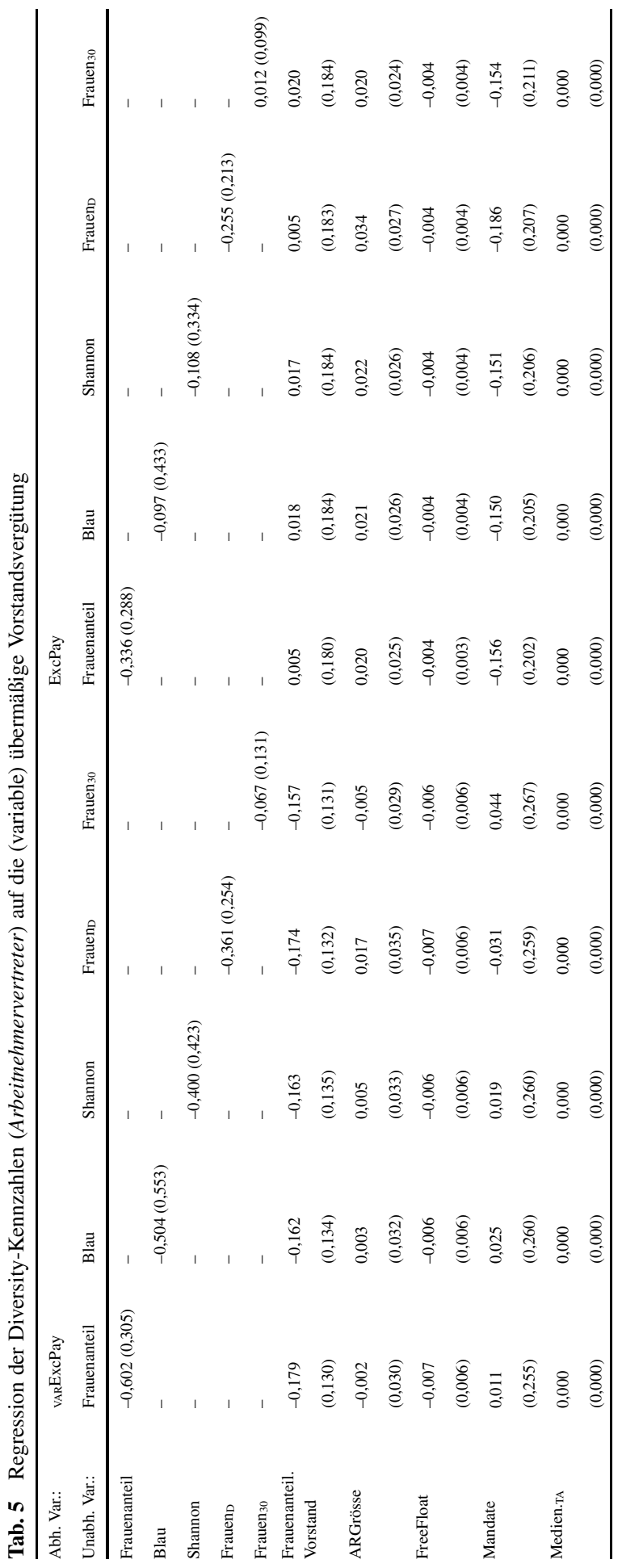




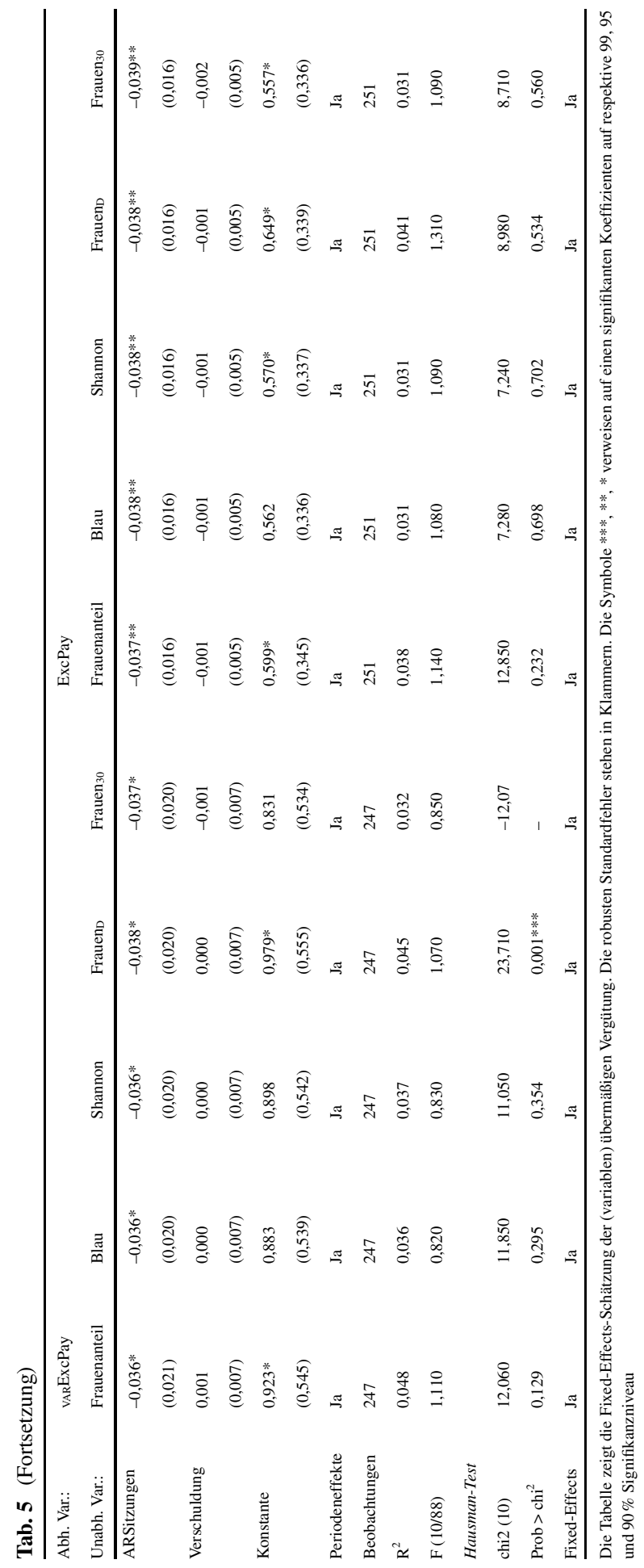




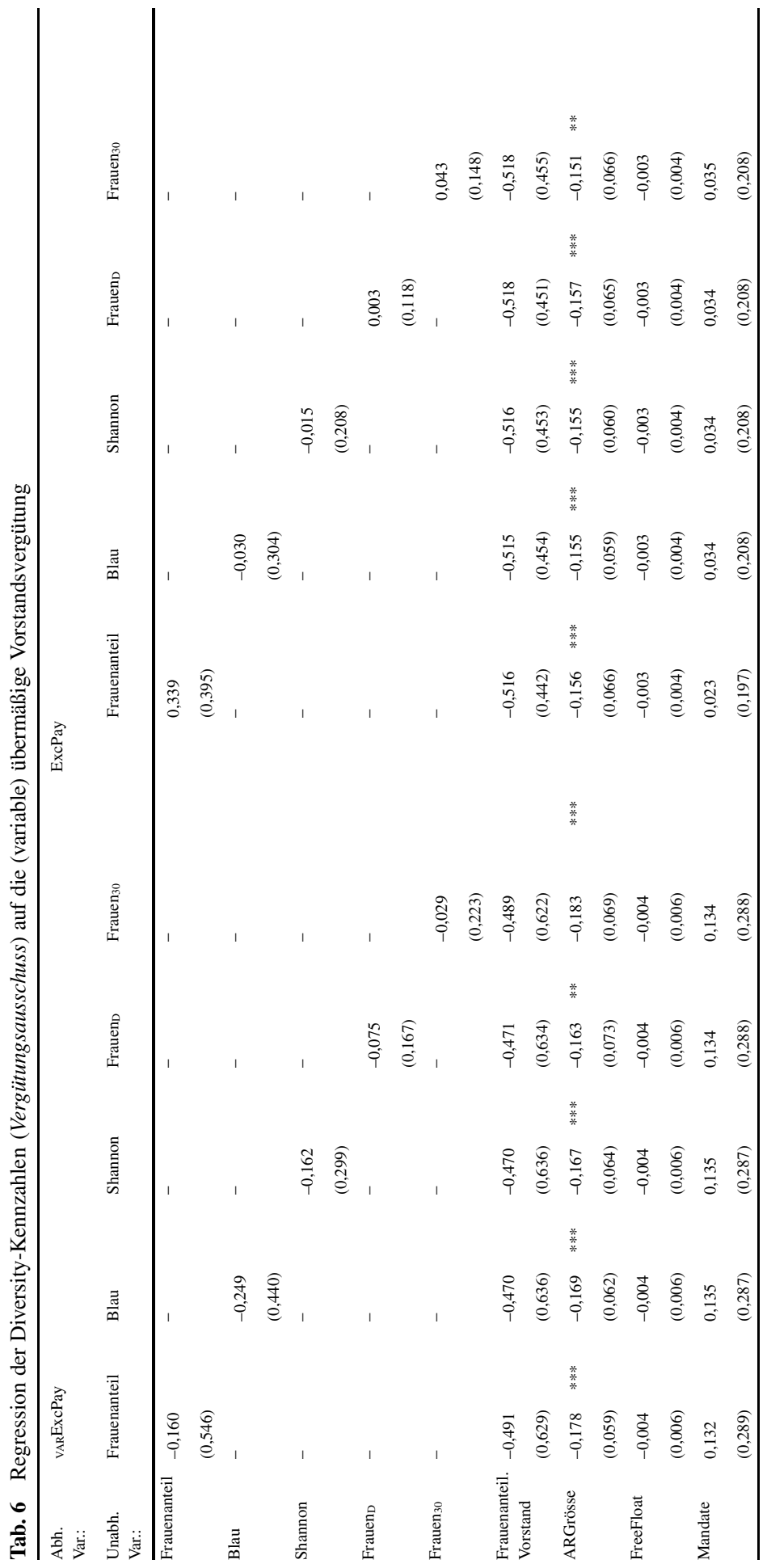




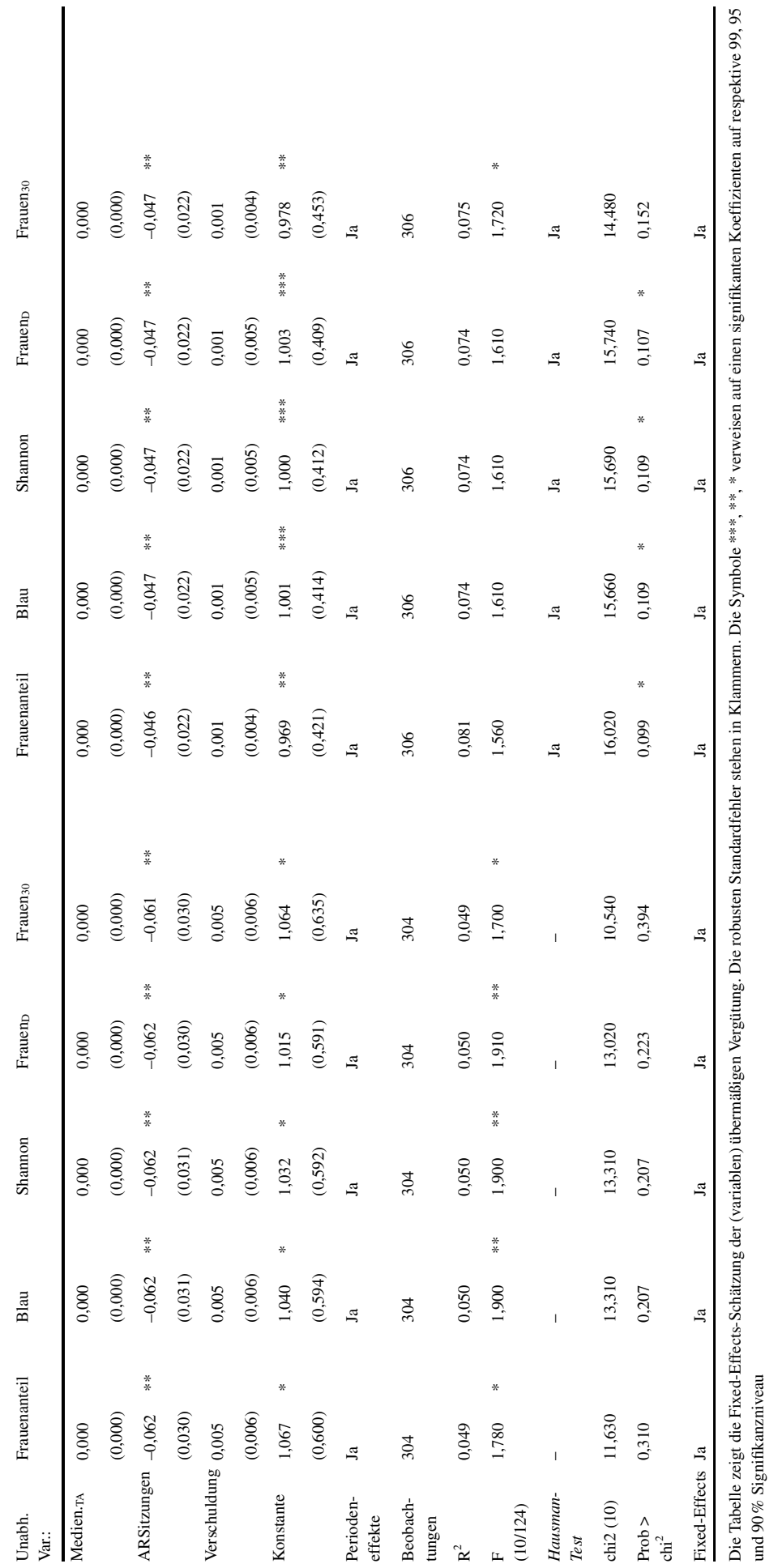


weisen für die unabhängigen Variablen $\beta$-Koeffizienten im Intervall von $-0,602$ bis $-0,067$ bzw. von $-0,336$ bis 0,012 aus (Tab. 5). Die Regressionen auf die abhängigen Variablen ${ }_{V A R} E x c P a y$ und ExcPay sind für die unabhängigen Variablen durchgehen statistisch nicht signifikant. Die Ergebnisse stützen die Vermutung, dass Arbeitnehmervertreter geringer in die Entscheidungsfindung involviert seien, da Beschlüsse regelmäßig in Ausschüssen vorbereitet werden, in denen Arbeitnehmervertreter seltener vertreten sind (Raabe 2011). Die Hypothese 3a ist somit zu verwerfen.

Entgegen der Untersuchungen zum gesamten Aufsichtsrat sowie den Aktionärsvertretern liefert das Modell zum Vergütungsausschuss keine signifikanten Befunde (Tab. 6). Die Analyse deutet somit darauf hin, dass zwischen der Frauenpräsenz im Ausschuss und der übermäßigen Vergütung des Vorstands kein Zusammenhang besteht. Somit wird die Hypothese 4 a verworfen. Dies ist möglichweise darauf zurückzuführen, dass die Repräsentanz weiblicher Ausschussmitglieder zu gering ist, um einen statistischen Zusammenhang aufdecken zu können (Momtaz 2014). Respektive liegt der durchschnittliche Frauenanteil im Aufsichtsrat bei 12\%, $61 \%$ der Ausschüsse wird durch ausschließlich Männer vertreten und in lediglich $11 \%$ bzw. 36 der Unternehmen sind mehr als eine Frau im Vergütungsausschuss vertreten. Das Ergebnis ist damit konvergent mit insbesondere älteren Untersuchungen, welche ebenfalls aufgrund des geringen Frauenanteils - keinen Zusammenhang mit verschiedenen Unternehmensparametern feststellen konnten (Randøy et al. 2006; Rose 2007).

\subsubsection{Nichtlinearer Zusammenhang zwischen Gender-Diversity und Überwachungseffektivität}

Die nachfolgend durchgeführten Regressionsanalysen untersuchen den Zusammenhang zwischen der absoluten Anzahl weiblicher Aufsichtsräte und der (variablen) übermäßigen Vorstandsvergütung. Anhand der vier bzw. drei Dummy-Variablen beobachten wir, wie sich die Stärke des vorgenannten Zusammenhangs bei einer, zwei, drei oder mindestens vier Frauen im Vergleich zu keiner Frau verändert. Die Ergebnisse der Analyse zeigen, dass - unabhängig von der Anzahl weiblicher Aufsichtsräte in einem Gremium - die abhängige Variable und unabhängigen Variablen in einem inversen Verhältnis zueinanderstehen. Die Schätzungen der gesamten und der variablen abnormalen Vergütung legen dabei konsistente Ergebnisse offen, die für die Aktionärsvertreter sowie den gesamten Aufsichtsrat, nicht aber für die Arbeitnehmervertreter und den Vergütungsausschuss signifikant sind. Den Ergebnissen entsprechend sinkt die übermäßige Vergütung umso stärker, je mehr Frauen im Kontrollgremium vertreten sind. Die $\beta$-Koeffizienten der binären Diversity-Variablen zeigen, dass die Stärke des Zusammenhangs mit zunehmender Frauenanzahl steigt (siehe Tab. 7). Diese Untersuchung demonstriert, dass es für die Arbeitsweise des Aufsichtsrats nicht allein relevant ist, ob eine Frau im Gremium präsent ist, sondern vielmehr wie viele Frauen vertreten sind. Vor diesem Hintergrund können wir die Hypothesen $1 \mathrm{~b}$ und $2 \mathrm{~b}$ bestätigen und müssen die Hypothesen $3 \mathrm{~b}$ und $4 \mathrm{~b}$ verwerfen.

Zur Veranschaulichung des Sachverhalts haben wir weitere Regressionen berechnet, in welchen wir die abnormale Vergütung durch lediglich die binären Diversity- 


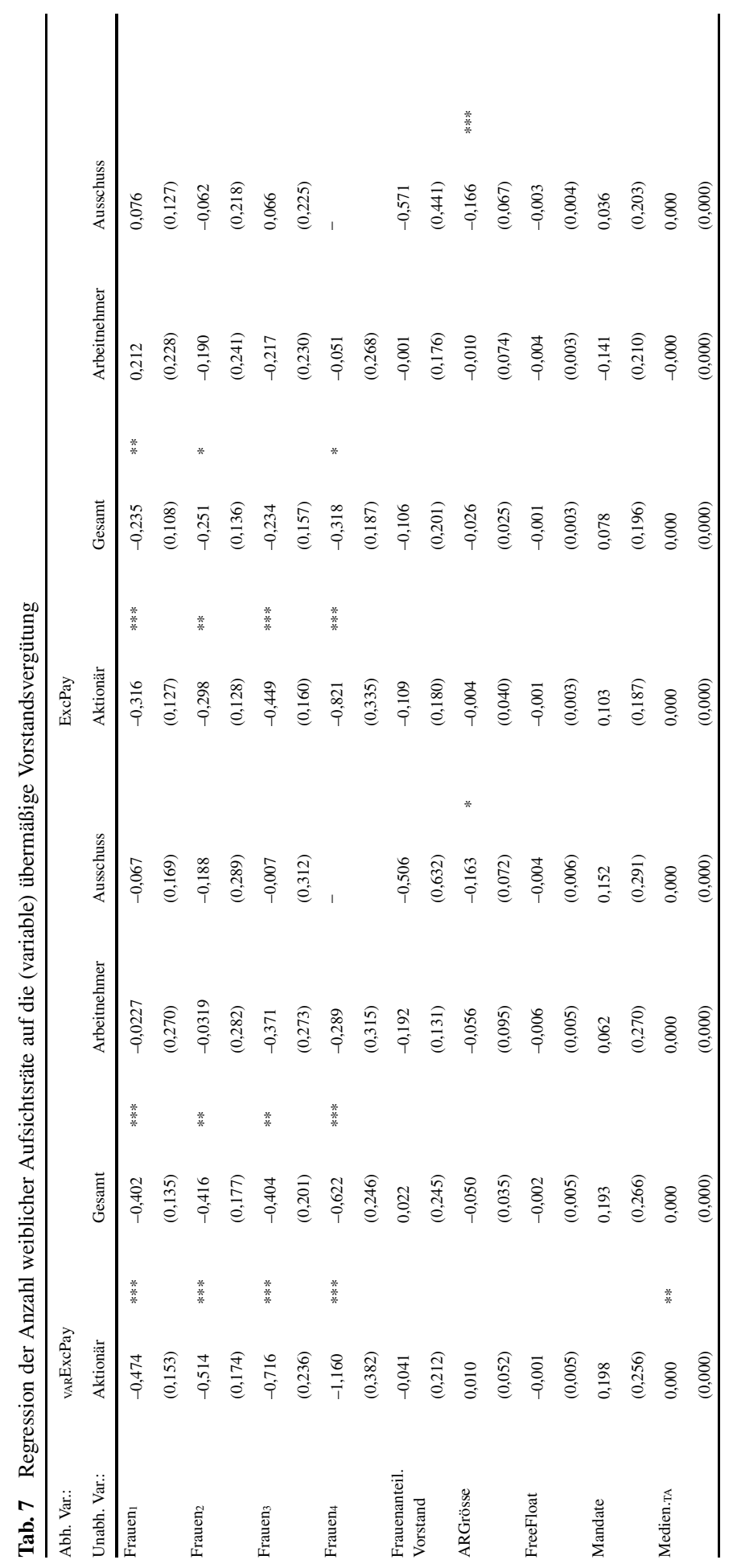




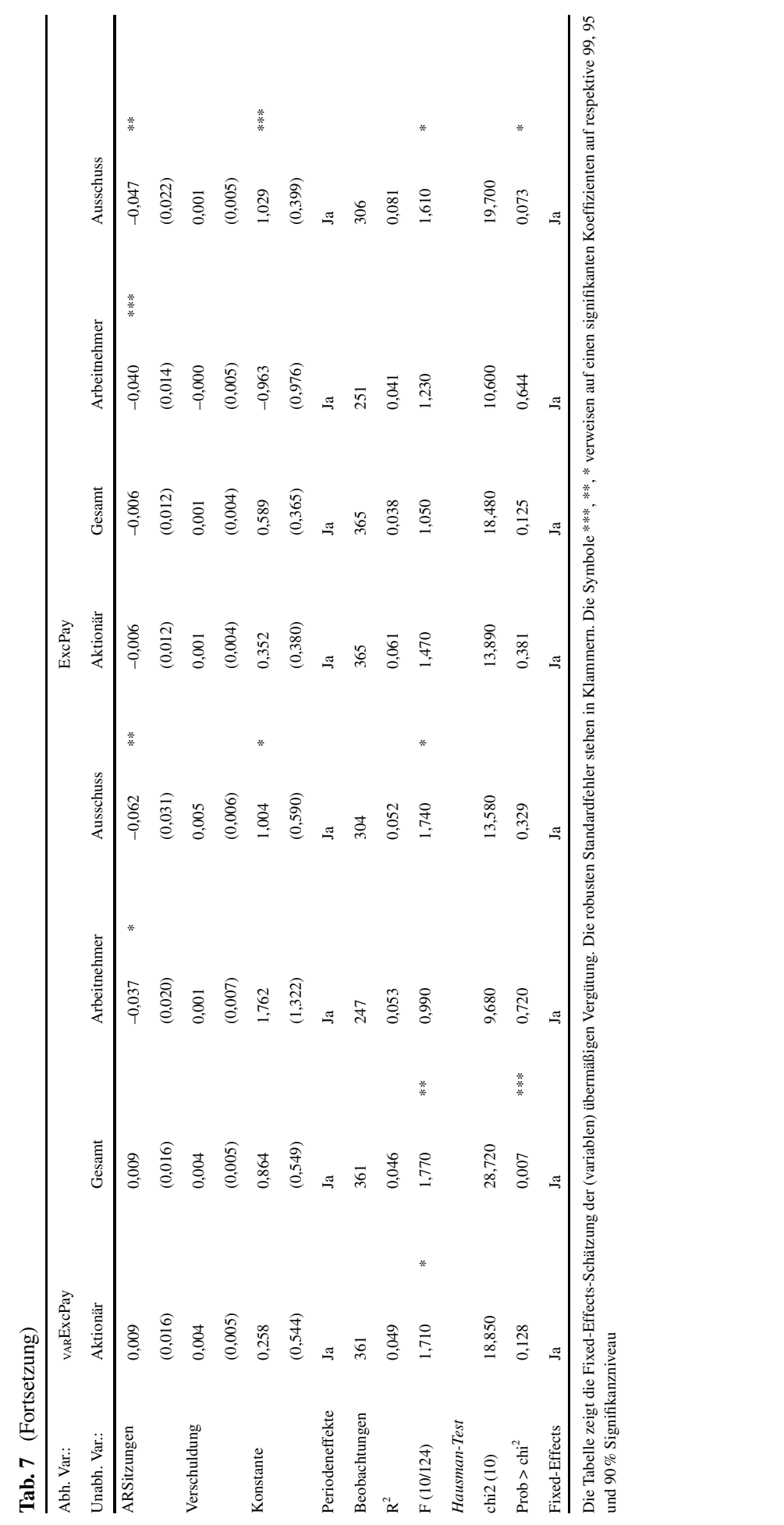




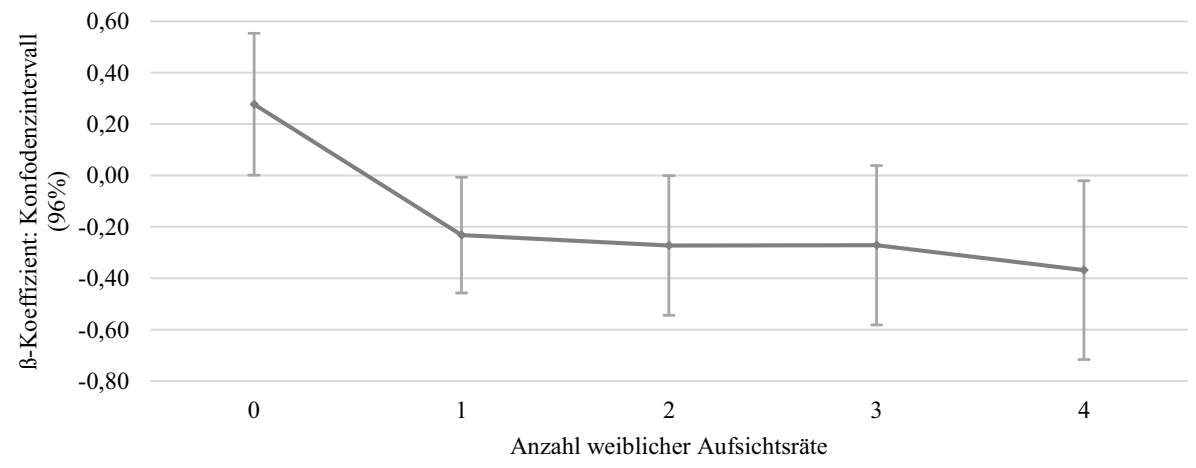

Abb. 2 Zusammenhang zwischen der Anzahl weiblicher Aufsichtsräte (gesamter Aufsichtsrat) und VARExcPay

Variablen schätzen und den Zusammenhang in Abb. 2 (exemplarisch für den gesamten Aufsichtsrat) präsentiert. Aus der Darstellung ist abzulesen, inwiefern die Stärke des Zusammenhangs bei einer zunehmenden Anzahl an Frauen variiert. Die Ergebnisse verdeutlichen, dass es für die Ausprägung der variablen übermäßigen

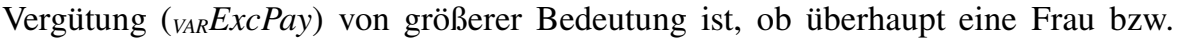
besonders viele Frauen im Aufsichtsrat vertreten sind. Die variable übermäßige Vergütung ist dagegen nahezu konstant, wenn zwei $\left(\right.$ Frauen $\left._{2}\right)$ statt nur einer Frau bzw. drei statt zwei Frauen (Frauen ${ }_{3}$ ) dem Gremium angehören. Dieser Effekt wird anhand des sprunghaften Abfalls der $\beta$-Koeffizienten von Frauen $_{0}$ (= Konstante) auf Frauen $_{1}\left[\Delta \beta_{\text {Frauen1-0 }}\left(\right.\right.$ VARExCPay $\left._{2}=-0,510\right]$ sowie von Frauen 3 auf Frauen 4 [ $\left[\Delta \beta_{\text {Frauen4-3 }}\right.$ $(\mathrm{vARExcPay})=-0,097]$ erkennbar. Damit stehen unsere Resultate lediglich teilweise im Einklang mit der Critical Mass Theorie. Sie entsprechen jedoch den Erkenntnissen der Studie von Usman et al. (2018), welche den Zusammenhang zwischen weiblichen Aufsichtsräten im Vergütungsausschuss und der CEO-Vergütung empirisch betrachtet. Während die Critical Mass Theorie proklamiert, dass Gruppen mit ausschließlich männlichen Mitgliedern (uniform groups) eine höhere Arbeitsleistung hervorbringen, als Gruppen mit sehr wenigen Frauen (skewed groups), zeigen unsere Ergebnisse, analog zu Usman et al. (2018), dass Aufsichtsräte mit mindestens einer Frau eine bessere Überwachungseffektivität aufweisen, als Überwachungsorgane ohne weibliche Mitglieder. Es ist also davon auszugehen, dass der Beintritt einer Frau in den Aufsichtsrat einen maßgeblichen Einfluss auf das Gruppengefüge und damit die Arbeitsweise des Gremiums hat. Die effektivsten Kontrolleure sind, unseren Befunden entsprechend, jedoch Aufsichtsräte, in denen mindestens vier Frauen (tilted groups) vertreten sind. Dieser kritische Wert von vier Aufsichtsrätinnen entspricht durchschnittlich einem Frauenanteil von 32\%. Anhand unseres Datensatz und der aktuellen Repräsentanz weiblicher Kontrolleure, lässt sich die Überwachungseffektivität von Aufsichtsräten mit einem ausgeglichenen Geschlechterverhältnis nicht überprüfen, da sehr wenige Überwachungsorgane einen Frauenanteil von über $40 \%$ bzw. 50\% aufweisen können. Insofern bleibt an dieser Stelle offen, ob balanced groups sogar die Arbeitsleistung der tilted groups überschreiten können und die Überwachungseffektivität ggf. weiterhin erhöht werden kann. 


\subsubsection{Robustheitsüberprüfung}

Mit dem Ziel die Robustheit der Ergebnisse zu überprüfen, variieren wir die abhängige Variable unseres Regressionsmodells. Dazu ersetzten wir die übermäßige

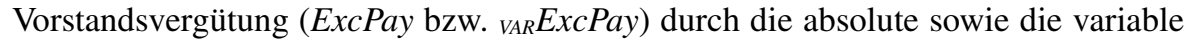
pro Kopf Vergütung des Vorstands (totalPay bzw. VARPay). Die Ergebnisse identifizieren ebenfalls einen negativen und überwiegend signifikanten Zusammenhang zwischen den Diversity-Variablen der Aktionärsvertreter sowie des gesamten Aufsichtsrats und der (variablen) Vorstandsvergütung. Die Regression der DiversityKennzahlen des Vergütungsausschusses auf die Vorstandsvergütung führt weiterhin zu insignifikanten Ergebnissen (nicht tabuliert). Wir können somit von robusten Ergebnissen ausgehen. Zudem zeigte sich bereits im Grundmodell unserer Schätzung, dass unsere Ergebnisse über verschiedenen Diversity-Kennzahlen hinweg konstant sind.

\section{Fazit und Ausblick}

Die vorliegende Studie liefert einen Beitrag über die Repräsentanz sowie den theoretischen und empirischen Einfluss von Frauen im Aufsichtsrat deutscher börsennotierter Unternehmen. Aus dem Kontext der jüngst eingeführten und regelmäßig diskutierten gesetzlichen Geschlechterquote liefern wir neue Erkenntnisse zur ökonomischen Wirkung dieser Vorschrift. Unser Datensatz legte dabei offen, dass der Frauenanteil in den Aufsichtsräten deutscher börsennotier Gesellschaften in den letzten Jahren (2015-2017) sukzessive gestiegen ist, jedoch insbesondere in den kleineren Unternehmen (SDAX) und in Hinblick auf die Position des Aufsichtsratsvorsitzes weiterer Förderung bedarf. Auf Grundlage der Prinzipal Agenten Theorie und Resource Dependence Theorie sowie mehrerer unternehmensfixer Panelregressionen, haben wir den theoretischen und empirischen Zusammenhang von Frauen im Aufsichtsrat und der Überwachungseffektivität des Gremiums untersucht. Vor dem Hintergrund der theoretischen Ansätze und nationalen Vorschriften zur Vorstandsvergütung ergibt sich, dass die Höhe der Vergütung durch bspw. die Unternehmensgröße und -performance, Wachstumsmöglichkeiten sowie Branchenzugehörigkeit etc. zu erklären ist und eine hierüber hinausgehende Vergütung (excess pay) nicht existieren sollte (Winkler und Behrmann 2019). Die Überwachungseffektivität approximieren wir anhand der (variablen) übermäßigen Vorstandsvergütung, die den Anteil der Vergütung erfasst, der nicht durch ökonomische Determinanten erklärt werden kann und somit die Ineffizienzen der Kontrolltätigkeit des Aufsichtsrats abbildet. Im Ergebnis demonstriert unsere Untersuchung, dass Gender-Diversity und die (variable) übermäßige Vorstandsvergütung positiv assoziiert sind. Der Zusammenhang ist umso stärker, wenn genau eine Frau im Aufsichtsrat vertreten ist oder ein Schwellenwert von vier Frauen überschritten wird. Diese Ergebnisse stehen teilweise im Einklang mit der Critical Mass Theorie. Entgegen der Theorie proklamieren unsere Befunde, dass sich selbst bei lediglich einer Frau im Aufsichtsrat die vergütungsbezogene Überwachungseffektivität des Kontrollorgans verbessert. Im Sinne der Critical Mass Theorie können wir auf Basis unserer Untersuchung davon ausgehen, dass - in 
Hinblick auf eine möglichst geringe übermäßige Vergütung - eine ausgeglichene Geschlechterrepräsentanz im Aufsichtsrat anzustreben ist. Die vorliegenden Ergebnisse stützen die Erkenntnisse der Studien von Adams und Ferreira (2009), LucasPérez et al. (2015) sowie Baixauli-Soler et al. (2016), die einen vorteilhaften Effekt von Frauen auf die Überwachungseffektivität vermuten. Daraus schließen wir, dass sich höhere Gender-Heterogenität, durch Erweiterung des Kompetenzspektrums des Aufsichtsrats, zu einer besseren Überwachung des Vorstands und somit einer höheren Corporate Governance-Qualität des Unternehmens führt. Mit Hinblick auf die Wirksamkeit der Geschlechterquote legen unsere Ergebnisse die Vermutung nahe, dass diese nicht auf Kosten guter Corporate Governance und effektiver Überwachung durch den Aufsichtsrat eingeführt wurde. Auf unseren Befunden aufbauend lassen sich folgende Implikationen und Forschungsansätze ableiten. Die Ergebnisse sprechen dafür, dass der Aufsichtsrat bei der Planung seiner Zusammensetzung auf eine gewisse Heterogenität seiner Mitglieder achten sollte. Diese begünstigt wiederum die Breite seines Ressourcenpools und schlägt sich vorteilhaft auf seine Arbeitsweise nieder. Eine reine Befolgung der Quote (bspw. mittels sog. „Goldröcke“) dürfte jedoch, vor allem in Zeiten von Nachhaltigkeitstrends (Gender-Diversity als Teil des CSR-Reporting) und des gesellschaftlichen Wandels, zu einer negativen öffentlichen Wahrnehmung und Unverständnis führen. In diesem Kontext und in Hinblick auf weitere Forschungsansätze kann von Interesse sein, inwiefern bei der Besetzung vakanter Aufsichtsratspositionen primär weibliche Aufsichtsräte berücksichtigt werden, die bereits in anderen Kontrollgremien vertreten sind (Oehmichen et al. 2010). Zukünftige Forschung könnte vor diesem Hintergrund untersuchen, inwiefern die Geschlechterquote den Umfang von Mehrfachmandaten von Aufsichtsrätinnen (weitere Mandate in Leitungs- oder Überwachungsgremium in rechtlich unabhängigen Unternehmen) determiniert (Handschumacher et al. 2019) und in diesem Zusammenhang hinterfragen, ob die Geschlechterquote tatsächlich mehr Frauen Chancen auf eine Aufsichtsratsposition ermöglicht oder sich der Frauenanteil lediglich durch Mehrfachmandatierung einiger weniger Aufsichtsrätinnen erhöht.

Funding Open Access funding provided by Projekt DEAL.

Open Access Dieser Artikel wird unter der Creative Commons Namensnennung 4.0 International Lizenz veröffentlicht, welche die Nutzung, Vervielfältigung, Bearbeitung, Verbreitung und Wiedergabe in jeglichem Medium und Format erlaubt, sofern Sie den/die ursprünglichen Autor(en) und die Quelle ordnungsgemäß nennen, einen Link zur Creative Commons Lizenz beifügen und angeben, ob Änderungen vorgenommen wurden.

Die in diesem Artikel enthaltenen Bilder und sonstiges Drittmaterial unterliegen ebenfalls der genannten Creative Commons Lizenz, sofern sich aus der Abbildungslegende nichts anderes ergibt. Sofern das betreffende Material nicht unter der genannten Creative Commons Lizenz steht und die betreffende Handlung nicht nach gesetzlichen Vorschriften erlaubt ist, ist für die oben aufgeführten Weiterverwendungen des Materials die Einwilligung des jeweiligen Rechteinhabers einzuholen.

Weitere Details zur Lizenz entnehmen Sie bitte der Lizenzinformation auf http://creativecommons.org/ licenses/by/4.0/deed.de.

Interessenkonflikt F. Handschumacher und W. Ceschinski geben an, dass kein Interessenkonflikt besteht. 


\section{Literatur}

Abdullah, Shamsul N., Ku Nor Izah Ku Ismail, und Lilac Nachum. 2016. Does having women on boards create value? The impact of societal perceptions and corporate governance in emerging markets. Strategic Management Journal 37:466-476.

Adams, Renée B., und Daniel Ferreira. 2009. Women in the boardroom and their impact on governance and performance. Journal of Financial Economics 94:291-309.

Ahern, Kenneth R., und Amy K. Dittmar. 2012. The changing of the boards: the impact on firm valuation of mandated female board representation. The Quarterly Journal of Economics 127:137-197.

Aldamen, Husam, Janice Hollidale, und Jennifer L. Ziegelmayer. 2018. Female audit committee members and their influence on audit fees. Accounting and Finance 58:57-89.

Arnegger, Martin, und Christian Hoffmann. 2014. Überprüfung des Zusammenhangs von Eigenschaften, Aufgaben und Vergütung von Aufsichtsräten deutscher Unternehmen. Zeitschrift für betriebswirtschaftliche Forschung 66:518-566.

Babcock, Lina, und Sara Laschever. 2003. Women don't ask. Negotiation and the gender divide. Princeton: Princeton University Press.

Baixauli-Soler, J. Samuel, M. Encarnacion Lucas-Pérez, Juan Francisco Martin-Ugedo, Antonio MinguezVera, und Gregorio Sanchez-Marin. 2016. Executive directors' compensation and monitoring: the influence of gender diversity on Spanish boards. Journal of Business Economics and Management 17:1133-1154.

Bear, Stephen, Noushi Rahman, und Corinne Post. 2010. The impact of board diversity and gender composition on corporate social responsibility and firm reputation. Journal of Business Ethics 97:207-221.

Benkraiem, Ramzi, Amal Hamrouni, Faten Lakhal, und Nadia Toumi. 2017. Board independence, gender diversity and CEO compensation. Corporate Governance: The international Journal of Business in Society 17:845-860.

Boerner, Sabine, Hannah Keding, und Hendrik Hüttermann. 2012. Gender Diversity und Organisationserfolg - Eine kritische Bestandsaufnahme. Zeitschrift für betriebswirtschaftliche Forschung 64:37-70.

Börsch-Supan, Axel, und Jens Köke. 2002. An applied Econometricians' view of empirical corporate governance studies. German Economic Review 3:295-326.

Boulouta, Ioanna. 2013. Hidden connections: the link between board gender diversity and corporate social performance. Journal of Business Ethics 113:185-197.

Boyd, Brian K. 1990. Corporate linkages and organizational environment: A test of the resource dependence model. Strategic Management Journal 11:419-430.

Boyd, Brian K. 1994. Board control and CEO compensation. Strategic Management Journal 15:335-344.

Bozhinov, Viktor, Christopher Koch, und Thorsten Schank. 2017. Has the push for equal gender representation changed the role of women on German supervisory boards? Social Science Research Network. https://doi.org/10.2139/ssrn.3043760. Zugegriffen: 24. August 2019.

Bozhinov, Viktor, Christopher Koch, und Thorsten Schank. 2019. The second glass ceiling: women's role in supervisory boards of German firms. Schmalenbach Business Review 71:385-411.

Brunner, Markus. 2009. Resource-Dependence-Ansatz. In Theorien und Methoden der Betriebswirtschaft, Hrsg. M. Schwaiger, A. Meyer, 29-40. München: Vahlen.

Bugeja, Martin, Zoltan Matolcsy, und Helen Spiropoulos. 2016. The association between gender-diverse compensation committees and CEO compensation. Journal of Business Ethics 139:375-390.

Campbell, Kevin, und Antonio Mínguez-Vera. 2008. Gender diversity in the boardroom and firm financial performance. Journal of Business Ethics 83:435-451.

Carter, David A., Betty J. Simkins, und W. Gary Simpson. 2003. Corporate governance, board diversity, and firm value. The Financial Review 38:33-53.

Carter, Mary Ellen, Lei Li, Alan J. Marcus, und Hassan Tehraniana. 2016. Excess pay and deficient performance. Review of Financial Economics 30:1-10.

Chapple, Larelle, und Jacquelyn E. Humphrey. 2014. Does board gender diversity have a financial impact? Journal of Business Ethics 122:709-723.

Chen, Guoli, Craig Crossland, und Sterling Huang. 2016. Female board representation and corporate acquisition intensity. Strategic Management Journal 37:303-313.

Coles, Jeffrey L., D. Daniel Naveen, und Lalitha Naveen. 2014. Co-opted boards. The Review of Financial Studies 27:1751-1796.

Core, John E., Robert W. Holthausen, und David F. Larcker. 1999. Corporate Governance, chief executive officer compensation, and firm performance. Journal of Financial Economics 51:371-406. 
Cumming, Douglas, und Tak Yan Oliver Leung Rui. 2015. Gender Diversity and Securities Fraud. Academy of Management Journal 58:1572-1593.

Dienes, Dominik, und Patrick Velte. 2016. The impact of supervisory board composition on CSR reporting. Evidence from the German two-tier system. Sustainability 8:1-20.

Drobetz, Wolfgang, Andreas Schillhofer, und Heinz Zimmermann. 2004. Corporate governance and expected stock returns: evidence from Germany. European Financial Management 10:267-293.

Duran, Mihael, und Kerstin Pull. 2014. Der Beitrag der Arbeitnehmervertreter zur fachlichen und geschlechtlichen Diversität von Aufsichtsräten. Erkenntnisse aus einer qualitativ-explorativen Analyse. Industrielle Beziehungen 21:329-351.

Eulerich, Marc, und Carolin van Uum. 2017. Die Interne Revision als Unterstützer von Vorstand und Aufsichtsrat. Zeitschrift für betriebswirtschaftliche Forschung 69:275-310.

Eulerich, Marc, Christian Lohmann, Stefanie Haustein, und Dirk Tunger. 2014. Die Entwicklung der betriebswirtschaftlichen Corporate Governance-Forschung im deutschsprachigen Raum - Eine State of the Art-Analyse auf der Basis bibliometrischer Daten. Zeitschrift für betriebswirtschaftliche Forschung 66:567-600.

Faleye, Olubunmi, Rani Hoitash, und Udi Hoitash. 2011. The costs of intense board monitoring. Journal of Financial Economics 101:160-181.

Fama, Eugene F., und Michael C. Jensen. 1983. Separation of ownership and control. The Journal of Law and Economics 26:301-325.

Fehre, Kerstin, und Rebecca Spiegelhalder. 2017. Same same, but different: Eine Analyse des Humankapitals weiblicher und männlicher Aufsichtsräte in Deutschland. Zeitschrift für betriebswirtschaftliche Forschung 69:311-343.

Fessler, Thomas. 2013. Wirkung der Diversität von Aufsichtsräten auf die Unternehmensperformance in und nach der Finanzkrise: Eine Panelanalyse deutscher Aktiengesellschaften. München/Mehring: Hampp.

Freidank, Carl-Christian. 2012. Unternehmensüberwachung. Die Grundlagen betriebswirtschaftlicher Kontrolle, Prüfung und Aufsicht. München: Vahlen.

Gujarati, Damodar N., und Dawn C. Porter. 2010. Essentials of econometrics. Singapore: Irwin/McGrawHill.

Gulamhussen, Mohamed Azzim, und Sílvia Fonte Santa. 2015. Female directors in bank boardrooms and theirs influence on performance and risk-taking. Globale Finance Journal 28:10-23.

Handschumacher, Franziska, Maximilian Behrmann, Willi Ceschinski, und Anne-Kathrin Hinze. 2018. Auswirkungen der Aufsichtsrats-Diversity auf die Unternehmensperformance. Eine Bestandsaufnahme der empirischen Forschung im dualistischen System. Zeitschrift für Corporate Governance 13:124-130.

Handschumacher, Franziska, Maximilian Behrmann, Willi Ceschinski, und Remmer Sassen. 2019. Do board interlocks support monitoring effectiveness? Evidence from listed German companies. Management Research Review 42:1278-1296.

Harjoto, Maretno, Indrarini Laksmana, und Robert Lee. 2015. Board diversity and corporate social responsibility. Journal of Business Ethics 132:641-660.

Harrison, David A., und Katherine J. Klein. 2007. What's the difference? Diversity constructs as separation, variety, or disparity in organizations. Academy of Management Review 32:1199-1228.

Hassan, Rohail, und Maran Marimuthu. 2016. Corporate governance, board diversity, and firm value: examining large companies using panel data approach. Economics Bulletin 36:1737-1750.

Hausman, Jerry A. 1978. Specification tests in econometrics. Econometrica: Journal of the Econometric Society 46:1251-1271.

Hillman, Amy J., und Thomas Dalziel. 2003. Boards of directors and firm performance: Integrating agency and resource dependence perspectives. Academy of Management Review 28:383-396.

Hillman, Amy J., Albert A. Cannella, und Ramona L. Paetzold. 2000. The resource dependence role of corporate directors: strategic adaptation of board composition in response to environmental change. Journal of Management Studies 37:235-256.

Hochhold, Stefanie, und Bernd Rudolph. 2009. Principal-Agent-Theorie. In Theorien und Methoden der Betriebswirtschaft, Hrsg. M. Schwaiger, A. Meyer, 131-145. München: Vahlen.

Hutchinson, Marion, Janet Mack, und Kevin Plastow. 2015. Who selects the 'right' directors? An examination of the association between board selection, gender diversity and outcomes. Accounting \& Finance 55:1071-1103.

Ittonen, Kim, Johanna Miettinen, und Sami Vähämaa. 2010. Does female representation on audit committees affect audit fees? Quarterly Journal of Finance and Accounting 49:113-139. 
Jensen, Michael C., und William H. Meckling. 1976. Theory of the firm: managerial behavior, agency costs and ownership structure. Journal of Financial Economics 3:305-360.

Joecks, Jasmin, Kerstin Pull, und Karin Vetter. 2013. Gender diversity in the boardroom and firm performance. What exactly constitutes a "critical mass"? Journal of Business Ethics 118:61-72.

John, Kose, und Lemma W. Senbet. 1998. Corporate governance and board effectiveness. Journal of Banking \& Finance 22:371-403.

Kanter, Rosabeth Moss. 1977. Some effects of proportions on group life. Shewed sex rations and responses to token women. American Journal of Sociology 82:965-990.

Kara, Michael. 2010. Vorstandsvergütung in der deutschen Corporate Governance. Eine ökonomische Analyse der Vorstandsvergütung am Beispiel der DAX 30 Unternehmen. Düsseldorf: Düsseldorf University Press.

Kirsch, Anja. 2018. The gender composition of corporate boards: a review and research agenda. The Leadership Quarterly 29:346-364.

Konrad, Alison M., Vicki Kramer, und Sumru Erkut. 2008. Critical mass: the impact of three or more women on corporate boards. Organization Dynamics 37:145-164.

Lenard, Mary Jane, Bing Yu, E. Anne York, und Shengxiong Wu. 2014. Impact of board gender diversity on firm risk. Managerial Finance 40:787-803.

Lucas-Pérez, Maria Encarnación, Antonio Mínguez-Vera, Juan Samuel Baixauli-Soler, Juan Francisco Martín-Ugedo, und Gregorio Sánchez-Marín. 2015. Women on the board and managers' pay: evidence from Spain. Journal of Business Ethics 129:265-280.

Maaß, Katja. 2016. Frauenquote oder das Märchen von der Gleichberechtigung. In Chefsache Frauenquote, Hrsg. P. Buchenau, 99-120. Wiesbaden: Springer Gabler.

Manita, Riadh Maria G.Bruna, Rey Dang, und L. Hocine Houanti. 2018. Board gender diversity and ESG disclosure: evidence from the USA. Journal of Applied Accounting Research 19:206-224.

von Meyerinck, Felix, Alexandra Niessen-Ruenzi, Markus Schmid, und Solomon Davidoff. 2020. As California goes, so goes the nation? The impact of board gender quotas on firm performance and the director labor market. Social Science Research Network https://doi.org/10.2139/ssrn.3303798. Zugegriffen: 15. Mai 2020.

Momtaz, Peyman. 2014. Beeinflusst die Geschlechterquote den Unternehmenserfolg? Bestandsaufnahme der Geschlechterquote in Aufsichtsräten und Vorständen sowie Einflussanalyse für den Unternehmenserfolg. Zeitschrift für Corporate Governance 9:249-254.

Nicholson, Gavin J., und Geoffrey C. Kiel. 2007. Can directors impact performance? A case-based test of three theories of corporate governance. Corporate Governance: An international Review 15:585-608.

Oehmichen, Jana. 2011. Mehrfachmandate von Aufsichtsratsmitgliedern. Eine Panel-Analyse ihrer Wirkung in deutschen Unternehmen. München/Mering: Hampp.

Oehmichen, Jana, Marc Steffen, und Michael Wolff. 2010. Der Einfluss der Aufsichtsratszusammensetzung auf die Präsenz von Frauen in Aufsichtsräten. Zeitschrift für betriebswirtschaftliche Forschung 62:503-532.

Perryman, Alexa A., Guy Fernando, und Arindam Tripathy. 2016. Do gender differences persist? An examination of gender diversity on firm performance, risk, and executive compensation. Journal of Business Research 69:579-586.

Pfeffer, Jeffrey, und Gerald R. Salancik. 1978. The external control of organizations: a resource dependence perspective. New York: Harper \& Row.

Prinz, Enrico. 2012. Der Einfluss von Mehrfachmandatsträgern auf die Unternehmensperformance. Zeitschrift für Betriebswirtschaft 82:875-911.

Proppe, Dennis. 2009. Endogenität und Instrumentenschätzer. In Methodik der empirischen Forschung, Hrsg. S. Albers, D. Klapper, U. Konradt, A. Walter, und J. Wolf, 253-282. Wiesbaden: Gabler.

Raabe, Nico. 2011. Mitbestimmung im Aufsichtsrat: Theorie und Wirklichkeit in deutschen Aktiengesellschaften. Berlin: Erich Schmidt Verlag.

Randøy, Trond, Steen Thomsen, und Lars Oxelheim. 2006. A nordic perspective on corporate board diversity. working paper, nordic innovation center. http://www.diva-portal.org/smash/get/diva2:707100/ FULLTEXT02. Zugegriffen: 20. Mai 2020.

Rapp, Marc, und Michael Wolff. 2010. Determinanten der Vorstandsvergütung. Zeitschrift für Betriebswirtschaft 80:1075-1112.

Rapp, Marc Steffen, Marco O. Sperling, und Michael Wolff. 2010. Who is asking the shareholders? Voting on management compensation in German listed firms - evidence from the annual meeting season 2010. HHL Research Paper Series in Corporate Governance. https://doi.org/10.2139/ssrn.1723155. Zugegriffen: 23. Juli 2019. 
Reguera-Alvarado, Nuria, Pilar de Fuentes, und Joaquina Laffarga. 2017. Does board gender diversity influence financial performance? Evidence from Spain. Journal of Business Ethics 141:337-350.

Reinwald, Max, Hendrik Hüttermann, Julia Kröll, und Sabine Boerner. 2015. Gender Diversity in Führungsteams und Unternehmensperformance: Eine Mataanalyse. Zeitschrift für betriebswirtschaftliche Forschung 67:262-296.

Rose, Caspar. 2007. Does female board representation influence firm performance? The Danish evidence. Corporate Governance: An International Review 15:404-413.

Schmidt, Sascha, und Matthias Brauer. 2005. Corporate Governance: Möglichkeiten des Aufsichtsrats zur sachgerechten Kontrolle von Managerverhalten und Firmenpolitik. Industrielle Beziehungen: Zeitschrift für Arbeit, Organisation und Management 12:306-322.

Schneider, Holger. 2009. Nachweis und Behandlung von Multikollinearität. In Methodik der empirischen Forschung, Hrsg. S. Albers, D. Klapper, U. Konradt, A. Walter, und J. Wolf, 221-236. Wiesbaden: Gabler.

Schulten, Axel. 2013. Rollenverständnis und Vergütung des deutschen Aufsichtsrats: eine empirische Analyse. Wiesbaden: Springer Gabler.

Seo, Jeongil. 2017. Board effectiveness and CEO pay: board information processing capacity, monitoring complexity, and CEO pay-for-performance sensitivity. Human Resource Management 56:373-388.

Shrader, Charles B., Virginia Blackburn, und Paul Iles. 1997. Women in management and firm financial performance: an exploratory study. Journal of Managerial Issues 9:355-372.

Sila, Vathunyoo, Angelica Gonzalez, und Jens Hagendorff. 2016. Women on board: does boardroom gender diversity affect firm risk? Journal of Corporate Finance 36:26-53.

Strobl, Sascha, Dasaratha V. Rama, und Suchismita Mishra. 2016. Gender diversity in compensation committees. Journal of Accounting, Auditing \& Finance 31:415-427.

Tebben, Tobias. 2011. Vergütungsanreize und opportunistische Bilanzpolitik: eine empirische Analyse der Rolle von Aufsichtsrat und Abschlussprïfer. Wiesbaden: Gabler.

Usman, Muhammad, Junrui Zhang, Fangjun Wang, Jungin Sun, und Muhammad A.M. Makki. 2018. Gender diversity in compensation committees and CEO pay: evidence from China. Management Decision 56:1065-1087.

Velte, Patrick. 2017a. Einfluss der Gender Diversity im Aufsichtsrat auf die externe Abschlussprüfung Eine empirische Untersuchung für den deutschen Kapitalmarkt (Teil A). Zeitschrift für Corporate Governance 12:219-224.

Velte, Patrick. 2017b. Einfluss der Gender Diversity im Aufsichtsrat auf die externe Abschlussprüfung Eine empirische Untersuchung für den deutschen Kapitalmarkt (Teil B). Zeitschrift für Corporate Governance 12:276-281.

Winkler, Ralf. 2018. Auswirkungen der Aufsichtsratsvergütung auf die Unternehmensüberwachung. Eine ökonometrische Studie zur Corporate Governance deutscher Aktiengesellschaften. Wiesbaden: Springer.

Winkler, Ralf, und Maximilian Behrmann. 2019. Aufsichtsratsvergütung und Überwachungseffektivität: Empirische Befunde für börsennotierte Gesellschaften in Deutschland. Zeitschrift für betriebswirtschaftliche Forschung 71:381-414. 